\title{
Psilocybin for End-of-Life Anxiety Symptoms: A Systematic Review and Meta-Analysis
}

\author{
Chia-Ling Yu', Fu-Chi Yang ${ }^{2,3}$, Szu-Nian Yang ${ }^{4,5,6}$, Ping-Tao Tseng ${ }^{7}$, Brendon Stubbs ${ }^{8,9,10}$, \\ Ta-Chuan Yeh ${ }^{11}$, Chih-Wei Hsu' ${ }^{12}$, Dian-Jeng Li ${ }^{13,14} \otimes$, and Chih-Sung Liang ${ }^{3,4} \bowtie$ \\ ${ }^{1}$ Department of Pharmacy, Chang-Gung Memorial Hospital, Linkou, Taiwan \\ ${ }^{2}$ Department of Neurology, Tri-Service General Hospital, National Defense Medical Center, Taipei, Taiwan \\ ${ }^{3}$ Graduate Institute of Medical Sciences, National Defense Medical Center, Taipei, Taiwan \\ ${ }^{4}$ Department of Psychiatry, Beitou Branch, Tri-Service General Hospital, National Defense Medical Center, Taipei, Taiwan \\ ${ }^{5}$ Department of Psychiatry, Armed Forces Taoyuan General Hospital, Taoyuan, Taiwan \\ ${ }^{6}$ Graduate Institute of Health and Welfare Policy, National Yang Ming University, Taipei, Taiwan \\ ${ }^{7}$ WinShine Clinics in Specialty of Psychiatry, Kaohsiung, Taiwan \\ ${ }^{8}$ Physiotherapy Department, South London and Maudsley NHS Foundation Trust, London, UK \\ ${ }^{9}$ Department of Psychological Medicine, Institute of Psychiatry, Psychology and Neuroscience (IoPPN), King's College London, \\ De Crespigny Park, London, UK \\ ${ }^{10}$ Positive Ageing Research Institute (PARI), Faculty of Health, Social Care and Education, Anglia Ruskin University, Chelmsford, UK \\ ${ }^{11}$ Department of Psychiatry, Tri-Service General Hospital, National Defense Medical Center, Taipei, Taiwan \\ ${ }^{12}$ Department of Psychiatry, Kaohsiung Chang Gung Memorial Hospital and Chang Gung University College of Medicine, Kaohsiung, Taiwan \\ ${ }^{13}$ Department of Addiction Science, Kaohsiung Municipal Kai-Syuan Psychiatric Hospital, Kaohsiung, Taiwan \\ ${ }^{14}$ Department of Nursing, Meiho University, Pingtung, Taiwan
}

Objective To systematically examine the effectiveness and tolerability of psilocybin for treating end-of-life anxiety symptoms.

Methods The Medline, Embase, CENTRAL, and PsycINFO databases were searched up to November 25, 2020. We enrolled clinical trials investigating psilocybin for treating end-of-life anxiety symptoms. Meta-analysis was conducted using random-effects model.

Results Overall, five studies were included, revealing that psilocybin was superior to the placebo in treating state anxiety at 1 day (Hedges' g, -0.70 ; 95\% confidence interval, -1.01 to -0.39$)$ and 2 weeks $(-1.03 ;-1.47$ to -0.60$)$ after treatment. Psilocybin was more effective than placebo in treating trait anxiety at 1 day $(-0.71 ;-1.15$ to -0.26$), 2$ weeks $(-1.08 ;-1.80$ to -0.36$)$, and 6 months $(-0.84 ;-1.37$ to $-0.30)$ after treatment. Psilocybin was associated with transient elevation in systolic $(19.00 ; 13.58-24.41 \mathrm{~mm} \mathrm{Hg})$ and diastolic $(8.66$; 5.18-12.15 mm Hg) blood pressure compared with placebo. The differences between psilocybin and placebo groups with regard to allcause discontinuation, serious adverse events, and heart rates were nonsignificant.

Conclusion Psilocybin-assisted therapy could ameliorate end-of-life anxiety symptoms without serious adverse events. Because of the small sample sizes of the included studies and high heterogeneity on long-term outcomes, future randomized controlled trials with large sample sizes are needed.

Psychiatry Investig 2021;18(10):958-967

Keywords Psilocybin; End-of-life anxiety; Life-threatening disease; Meta-analysis; Cancer.

\section{INTRODUCTION}

Patients with cancer might suffer from multidimensional psychological distress during diagnosis, treatment, and sur- vival. ${ }^{1,2}$ An epidemiological investigation involving 10,153 patients in Canada reported that $19.0 \%$ of patients had anxiety symptoms and $12.9 \%$ of them had clinical depression after a diagnosis of cancer. ${ }^{3}$ Notably, patients with cancer have a multi-

\footnotetext{
Received: June 17, 2021 Revised: July 15, 2021 Accepted: July 23, 2021

$\triangle$ Correspondence: Dian-Jeng Li, MD

Department of Addiction Science, Kaohsiung Municipal Kai-Syuan Psychiatric Hospital, No. 130, Kaisyuan 2nd Rd., Lingya Dist., Kaohsiung 802, Taiwan Tel: +886-7-751-3171, Fax: +886-7-16-1843, E-mail: edcrfvm45@hotmail.com

$\triangle$ Correspondence: Chih-Sung Liang, MD

Department of Psychiatry, Beitou Branch, Tri-Service General Hospital, National Defense Medical Center, No.60, Xinmin Road, Beitou District, Taipei 112, Taiwan Tel: +886-2-2895-9808, Fax: +886-222895-7633, E-mail: lcsyfw@gmail.com

(c) This is an Open Access article distributed under the terms of the Creative Commons Attribution Non-Commercial License (https://creativecommons.org/licenses/by-nc/4.0) which permits unrestricted non-commercial use, distribution, and reproduction in any medium, provided the original work is properly cited.
} 
dimensional etiology of mental health problems. The potentially life-threatening characteristic of cancer and the uncertainty related to its progression cancer might cause considerable psychological distress, ${ }^{4,5}$ with the fear of cancer recurrence and death also contributing to the stress. ${ }^{6}$ Recent cellular and molecular studies have identified that biological processes involving the immune and endocrine systems mediate the association between psychological distress and cancer progression. ${ }^{7,8}$

Similarly, patients with human immunodeficiency virus (HIV) might experience challenges related to the uncertainty regarding their future, which could induce significant psychological distress. ${ }^{9,10}$ Moreover, HIV-related stigma can contribute to poor mental health in these patients. ${ }^{11}$ The prevalence of anxiety disorders among patients with HIV has been estimated to be as high as $38 \%$, and these anxiety symptoms might impair adherence to antiretroviral treatment. ${ }^{12}$ Per the biological models, HIV infection in the central nervous system might induce neuroinflammatory reactions within the immune system, resulting in mental health problems. ${ }^{13}$ In summary, the complex etiologies of the mental health problems in patients with cancer or HIV can pose challenges for treatment.

Psilocybin-a substance obtained from psilocybin mushrooms-has been identified as a controlled substance in several countries including the United States to its dependence potential. ${ }^{14}$ It is a tryptamine serotoninergic psychedelic exerting its consciousness altering effects through $5-\mathrm{HT}_{2 \mathrm{~A}}$ agonism. ${ }^{15}$ Psilocybin is reported to produce highly spiritual states of consciousness with (up to 14-month) long-acting positive changes in cognition, affect, and behavior. ${ }^{16,17}$ In recent decades, psilocybin has garnered the researchers' interest in using it to treat several mental health problems, such as cancer-associated depression and anxiety ${ }^{18}$ and substance use disorders. ${ }^{19}$

Although psychopharmacological and psychosocial interventions are applied in treating anxiety in patients with cancer and HIV, the efficacy is mixed and limited..$^{20,21}$ Psilocybin has been shown to enhance spiritual wellbeing ${ }^{16,17}$ and ameliorate anxiety symptoms in patients with cancer. ${ }^{22}$ Therefore, in the palliative care and psycho-oncology disciplines, psilocybin can be considered a treatment option under controlled clinical practice for patients with cancer or HIV. Several clinical trials have explored the treatment efficacy of psilocybin for cancer-relat$\mathrm{ed}^{23}$ and HIV-related anxiety symptoms. ${ }^{24}$ Therefore, it would be helpful to summarize the treatment effectiveness and tolerability of psilocybin for use in patients with end-of-life anxiety symptoms.

The current study thus analyzed the effectiveness and tolerability of psilocybin in treating end-of-life anxiety symptoms in patients with cancer or HIV and explored its sustained effect on anxiety symptoms at different follow-up timepoints.

\section{METHODS}

\section{Database search}

This study was conducted per the guidelines of Preferred Reporting Items for Systematic Reviews and Meta-Analyses. Our search strategy is described in detail in the Supplementary Figure 1 (in the online-only Data Supplement). Two authors (Yu CL and Yang FC) independently performed electronic searches on the platforms, such as Medline, Embase, the Cochrane Central Register of Controlled Trials, and PsycINFO, up to November 25, 2020. At the initial stage, these two authors screened the titles and abstracts for eligibility through consensus. Subsequently, a third reviewer (Tseng PT) was consulted in situations where the former two authors could not achieve consensus.

\section{Inclusion and exclusion criteria}

The following eligibility criteria were applied; 1) peer-reviewed articles of clinical trials investigating the effectiveness of psilocybin in patients with life-threatening conditions, such as cancer and HIV;2) inclusion of both controlled studies (compared with a placebo) and uncontrolled studies (open-label single-arm); and 3) written in English language.

We excluded nonclinical trials, such as case reports and case series. Moreover, we excluded studies published as conference abstracts and those published in languages other than English.

\section{Primary and secondary outcomes}

The primary outcome was changes in anxiety symptoms, including state anxiety and trait anxiety. ${ }^{25}$ State anxiety reflects a transitory emotion characterized by heightened autonomic nervous system activity and consciously perceived feelings of tension, dread, and apprehension. By contrast, trait anxiety refers to a general tendency to respond with anxiety to perceived threats in the environment, and is a relatively stable characteristic of an individual. Individuals with high levels of trait anxiety would be more susceptible to stress, responding to several situations as if they were dangerous or threatening and demonstrating state anxiety reactions more frequently and with greater intensity than those with low trait anxiety.

The secondary outcomes were all-cause discontinuation; peak changes in heart rate (HR), systolic blood pressure (SBP), and diastolic blood pressure (DBP); and serious adverse events.

\section{Data extraction and quality appraisal}

Two authors (Yu CL and Liang CS) independently extracted data from the recruited studies. The GetData Graph Digitizer 2.26 was used to capture data from figures when necessary. In the cases of unavailable data in the literature, the corresponding authors were contacted and requested for the original data in 
at least two different occasions.

Two authors (CL Yu and DJ Li) independently assessed the quality of each included study. Disagreements were resolved through discussion with a third author (Liang CS). Moreover, to assess the quality of the included studies, we used the Cochrane risk-of-bias (ROB) tool, which consists of seven main domains (random sequence generation, allocation concealment, blindness of participants and personnel, blindness of outcome assessment, incomplete outcome data, selective reporting, and other source of bias). ${ }^{26}$

\section{Statistical analysis}

This meta-analysis was conducted using a random-effects model. Hedges' g with 95\% confidence interval (CI) was calculated for the primary outcomes. Negative values of Hedges' $g$ indicated anxiety score improvement. Weighted mean difference (WMD) was calculated for HR, SBP, and DBP. Odds ratio (OR) with 95\% CI was calculated for all-cause discontinuation. The time points of anxiety assessment were day 1 and month 1,3 , and 6 after single session of psilocybin treatment. In addition, the pre-post changes in the primary outcome at the last follow-up time point was examined for all included studies. Heterogeneity was examined using Cochran's Q test and using the $\mathrm{I}^{2}$ statistic. ${ }^{27}$ Notably, the scale of $\mathrm{I}^{2}$ ranges from $0 \%$ to $100 \%$, and values $>75 \%$ were considered having high heterogeneity. Meta-regression was not performed because $<10$ studies were included. Publication bias for primary outcomes was examined using funnel plots. In addition, trim-and-fill method was performed to estimate the number of unpublished studies and provide a summary effect adjusted for it. The threshold for statistical significance was set at a two-tailed $p$ value of $<0.05$ for all analyses.

\section{RESULTS}

\section{Selection protocol}

The details of search results are illustrated in Supplementary Table 1 (in the online-only Data Supplement). In summary, a total of 45 studies were selected at the stage of full-text review, of which 40 were excluded for various reasons. Overall, five articles met the inclusion criteria, and Table 1 summarizes their details.

\section{Characteristics and methodological quality of the included studies}

The mean sample size of the five included studies ${ }^{23,24,28-30}$ was 26.4 (standard deviation [SD]: 16.2; range: 12-56). The mean age was 54.4 (SD: 4.2 ) years, and the mean female proportion was $52.5 \%$ (SD: $29.8 \%$ ). All five studies provided the data on changes in state and trait anxiety symptoms. The time points of anxiety assessment were day 1 and month 1, 3, and 6 after a single session of psilocybin treatment.

Except for the blinding of outcome assessment, the quality of the other six domains were low or unclear ROBs for three RCTs $^{23,28,29}$ (Supplementary Figure 2 in the online-only Data Supplement). Considering all the five included studies (Supplementary Figure 3 in the online-only Data Supplement), the single-arm study by Anderson et al. ${ }^{24}$ was subjected to high ROB on random sequence generation, allocation concealment, blindness of participants and personnel, and blindness of outcome assessment. The post-RCT follow-up study by Agin-Liebes et al. ${ }^{30}$ was subjected to high ROB on blinding of outcome assessment.

Table 1. Characteristics and demographics of the included studies

\begin{tabular}{|c|c|c|c|c|c|c|c|c|c|}
\hline Study & Dx & $\begin{array}{l}\text { Sample } \\
\text { size }\end{array}$ & Female (\%) & Age & Dosing & Psychiatric comorbidities & Study design & Dropout & $\begin{array}{c}\text { Serious } \\
\mathrm{AE}\end{array}$ \\
\hline Grob et al. $^{28}$ & Cancer & 12 & 91.6 & $36-58$ & $\begin{array}{l}\text { Oral single dose, } \\
0.20 \mathrm{mg} / \mathrm{kg}\end{array}$ & $\begin{array}{l}\text { Yes. Specific psychiatric } \\
\text { disorders were not } \\
\text { mentioned }\end{array}$ & $\begin{array}{l}\text { Double-blind } \\
\text { RCT }\end{array}$ & 0 & No \\
\hline $\begin{array}{l}\text { Griffiths } \\
\text { et al. }^{23}\end{array}$ & Cancer & 56 & 49.0 & $56.3(10.0)$ & $\begin{array}{l}\text { Oral single dose, } \\
0.32 \mathrm{mg} / \mathrm{kg} \\
(22 \text { or } 30 \mathrm{mg})\end{array}$ & $\begin{array}{l}\text { Adjustment disorder; } \\
\text { dysthymia; GAD, } \\
\text { and MDD }\end{array}$ & $\begin{array}{l}\text { Double-blind } \\
\text { RCT }\end{array}$ & 5 & No \\
\hline Ross et al. ${ }^{29}$ & Cancer & 31 & 62.1 & $56.3(12.9)$ & $\begin{array}{l}\text { Oral single dose, } \\
0.3 \mathrm{mg} / \mathrm{kg}\end{array}$ & $\begin{array}{l}\text { Adjustment disorder } \\
\text { and GAD }\end{array}$ & $\begin{array}{l}\text { Double-blind } \\
\text { RCT }\end{array}$ & 3 & No \\
\hline 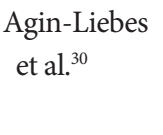 & Cancer & 15 & 60.0 & $53(13.5)$ & $\begin{array}{l}\text { Oral single dose, } \\
0.3 \mathrm{mg} / \mathrm{kg}\end{array}$ & $\begin{array}{l}\text { Adjustment disorder } \\
\text { and GAD }\end{array}$ & $\begin{array}{l}\text { Long-term } \\
\text { follow-up } \\
\text { after RCT }\end{array}$ & 1 & No \\
\hline $\begin{array}{l}\text { Anderson } \\
\text { et al. }\end{array}$ & $\begin{array}{l}\text { HIV/ } \\
\text { cancer }\end{array}$ & 18 & 0.0 & $59.2(4.4)$ & $\begin{array}{l}\text { Oral single dose, } \\
0.30-0.36 \mathrm{mg} / \mathrm{kg}\end{array}$ & $\begin{array}{l}\text { Mood disorder, anxiety } \\
\text { disorder, and } \\
\text { insomnia }\end{array}$ & $\begin{array}{l}\text { Open-label } \\
\text { single-arm } \\
\text { trial }\end{array}$ & 0 & No \\
\hline
\end{tabular}

$\mathrm{AE}$, adverse event; Dx, diagnosis; GAD, generalized anxiety disorder; MDD, major depressive disorder; RCT, randomized controlled trial 


\section{Primary outcome: changes in state and trait anxiety}

At day 1 after treatment (Figure 1), psilocybin was superior to placebo in treating state anxiety (Hedges' g, -0.70 ; 95\% CI, -1.01 to -0.39$)$ without significant heterogeneity $\left(\mathrm{I}^{2}=0.00 \%\right.$, Cochran's $\mathrm{Q}=1.48, \mathrm{p}=0.48$ ). A month later, the single session of psilocybin treatment was still superior to placebo in treating state anxiety (Hedges' g, -0.73 ; 95\% CI, -1.25 to -0.20 ) without significant heterogeneity $\left(\mathrm{I}^{2}=62.83 \%\right.$, Cochran's $\left.\mathrm{Q}=5.30, \mathrm{p}=0.07\right)$. However, the effect sizes of psilocybin versus placebo were not significant at 3-month (Hedges' g, -0.47 ; 95\% CI, -1.31 to 0.37 ; $\mathrm{I}^{2}=93.30 \%$ ) and 6-month (Hedges' $\mathrm{g},-0.88$; $95 \% \mathrm{CI},-1.78$ to 0.03 ; $\mathrm{I}^{2}=86.46 \%$ ) follow-ups. The study by Agin-Liebes et al..$^{30}$ assessed the state anxiety after 3.2 years and 4.5 years, revealing a Hedges' $g$ of -1.05 and -0.90 , respectively.

Figure 2 illustrates that at day 1 after treatment, psilocybin was superior to placebo in treating trait anxiety (Hedges' g, -0.71 ; $95 \%$ CI, -1.15 to -0.25$)$ without significant heterogeneity $\left(\mathrm{I}^{2}=\right.$ $39.22 \%$, Cochran's $\mathrm{Q}=3.43, \mathrm{p}=0.18$ ). One month later, psilocybin was still superior to placebo in treating trait anxiety (Hedges' g, $-1.04 ; 95 \%$ CI, -1.66 to -0.42 ) with significant moderate heterogeneity $\left(\mathrm{I}^{2}=65.66 \%\right.$, Cochran's $\left.\mathrm{Q}=6.66, \mathrm{p}=0.04\right)$. Notably, the effect size of psilocybin versus placebo was still significant at 3-month (Hedges' g, -0.60 ; 95\% CI, -1.16 to $-0.05 ; \mathrm{I}^{2}=84.87$ ) and 6-month (Hedges' $\mathrm{g}$, -1.03 ; 95\% CI, -1.54 to $-0.52 ; \mathrm{I}^{2}=68.76 \%$,) follow-ups; however, the heterogeneity was significant at both time points (month 3, $\mathrm{I}^{2}=84.87 \%, \mathrm{Q}=13.22, \mathrm{p}<0.001$; month 6, $\left.\mathrm{I}^{2}=68.76 \%, \mathrm{Q}=6.40, \mathrm{p}<0.001\right)$. The study by Agin-Liebes et al..$^{30}$ assessed the trait anxiety after 3.2 years and 4.5 years, revealing a Hedges' $g$ of -1.28 and -1.07 , respectively.

Figure 3 reveals the pre-post changes in state and trait anxiety symptoms at last follow-up time point for all included studies. Psilocybin was associated with significant changes in state (Hedges' g, -0.63 ; 95\% CI, -1.24 to -0.03 ) and trait (Hedges' g, -0.83 ; $95 \% \mathrm{CI},-1.25$ to -0.42 ) anxiety, whereas the heterogeneity was significant (both $\mathrm{p}<0.001$ ).

\section{Secondary outcome: effects on circulatory system and all-cause discontinuation}

Regarding the effects of psilocybin on the circulatory system (Figure 4), psilocybin revealed no differences compared with placebo regarding the $\mathrm{HR}$ (WMD, 2.85; 95\% CI, -4.51 to 10.20 ), and the heterogeneity was not significant $\left(\mathrm{I}^{2}=51.89 \%, \mathrm{Q}=4.15\right.$, $\mathrm{p}=0.13)$. However, psilocybin was significantly associated with elevation in SBP (WMD, 19.00; 95\% CI, 13.58 to 24.41) and DBP (WMD, 8.66; 95\% CI, 5.18 to 12.15 ) compared with the placebo. In addition, the heterogeneity was not significant for both SBP $\left(\mathrm{I}^{2}=8.92 \%, \mathrm{Q}=1.89, \mathrm{p}=0.39\right)$ and DBP $\left(\mathrm{I}^{2}=19.13 \%\right.$, $\mathrm{Q}=2.10, \mathrm{p}=0.35)$. All the included studies reported that the transient elevation in SBP and DBP was self-limited and required no medical intervention.
For all-cause discontinuation (Supplementary Figure 4 in the online-only Data Supplement), psilocybin did not differ from placebo (ORs: $1.53,95 \% \mathrm{CI}=0.37$ to 6.22 ) with nonsignificant heterogeneity $\left(\mathrm{I}^{2}=0.00 \%, \mathrm{Q}=0.09, \mathrm{p}=0.96\right)$.

\section{Publication bias and trim-and-fill analysis}

No small-study effects were observed (Supplementary Figures 5-14 in the online-only Data Supplement). The trim-andfill tests revealed that the effect sizes did not change the direction if there were imputed studies (Supplementary Figures 5-14 in the online-only Data Supplement).

\section{DISCUSSION}

\section{Main findings of the current study}

The current study comprehensively analyzed the effectiveness of psilocybin on different types of end-of-life anxiety (state and trait) at different time points after a single treatment session. The main findings revealed that psilocybin was superior to placebo in treating state anxiety after a single treatment session, with the effectiveness observed 1 day and 1 month after psilocybin treatment. In addition, psilocybin exhibited effectiveness in managing trait anxiety at the time points of 1 day and 1, 3, and 6 months after a single treatment session. Regarding the secondary outcomes, no serious adverse events were observed after single session of psilocybin treatment, and group difference related to all-cause discontinuation was not significant. Although psilocybin significantly affected the SBP and DBP, these effects were transient and self-limited and required no medical intervention.

Notably, trait anxiety has more long-term effects on patients than does state anxiety. Trait anxiety is associated with stress coping strategies and could be considered part of the personality dimension of neuroticism rather than emotional stability. ${ }^{25}$ A cross-sectional study enrolling nursing students reported that the greatest predictor of perceived stress was anxiety trait, and perceived stress was also correlated to several coping strategies, such as problem-solving. ${ }^{31}$ Among women with breast cancer, trait anxiety was significantly associated with problem-focused coping strategies with a focus on emotion. ${ }^{32}$ A study exploring personality parameters reported significant decreases in neuroticism scores after psilocybin therapy. ${ }^{33}$ Therefore, a sustained improvement in trait anxiety might indicate the benefits of psilocybin in improving stress coping strategies, which is crucial for patients with life-threatening illnesses who typically experience chronic stress.

Therefore, analyzing the short-term and long-term effectiveness of a single session psilocybin treatment on end-of-life anxiety is crucial in individuals with life-threatening illnesses. Most often these patients receive polypharmacy for their life-threaten- 


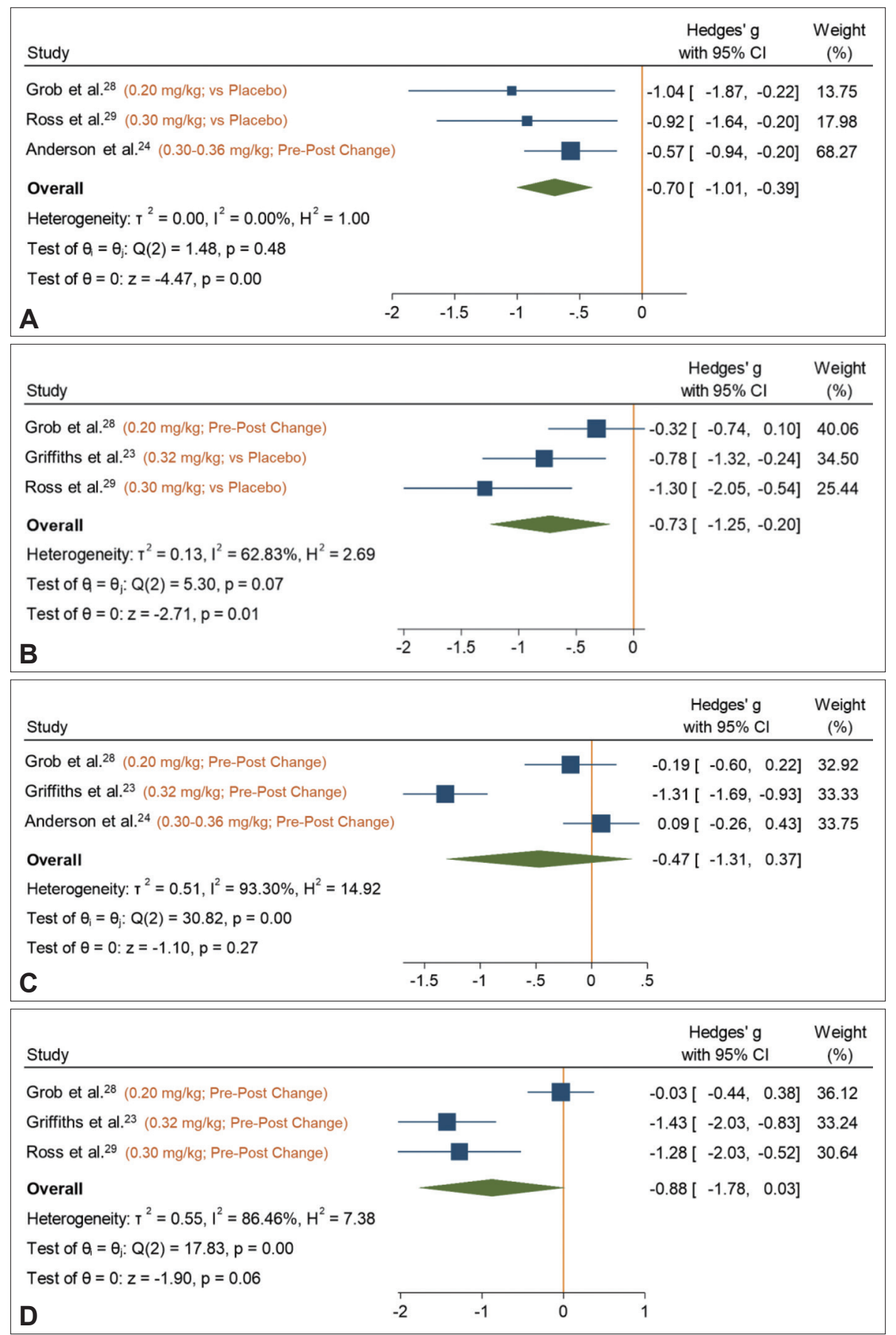

Figure 1. Changes in state anxiety after psilocybin treatment. A: Day 1. B: Month 1. C: Month 3. D: Month 6.

ing illnesses, such as chemotherapy or highly active antiretroviral therapy. Therefore, a single session of psilocybin treatment might reduce the risk of intolerance and drug-drug interaction. Notably, our study demonstrated that the effectiveness of a single psilocybin treatment session on trait anxiety could persist up to 6 months. Moreover, psilocybin exhibited merit in tolerability, revealing no significant differences in all-cause discontinuation and HR compared with placebo, and the effects on
SBP and DBP were transient and self-limiting. These promising findings regarding psilocybin use in end-of-life anxiety symptoms could prove helpful for clinicians in hospice care or psycho-oncology.

\section{Potential anxiolytic mechanisms of psilocybin}

Similar to the biomedical mechanism of psychedelics, psilocybin exerts psychoactive effects through the agonistic effects 


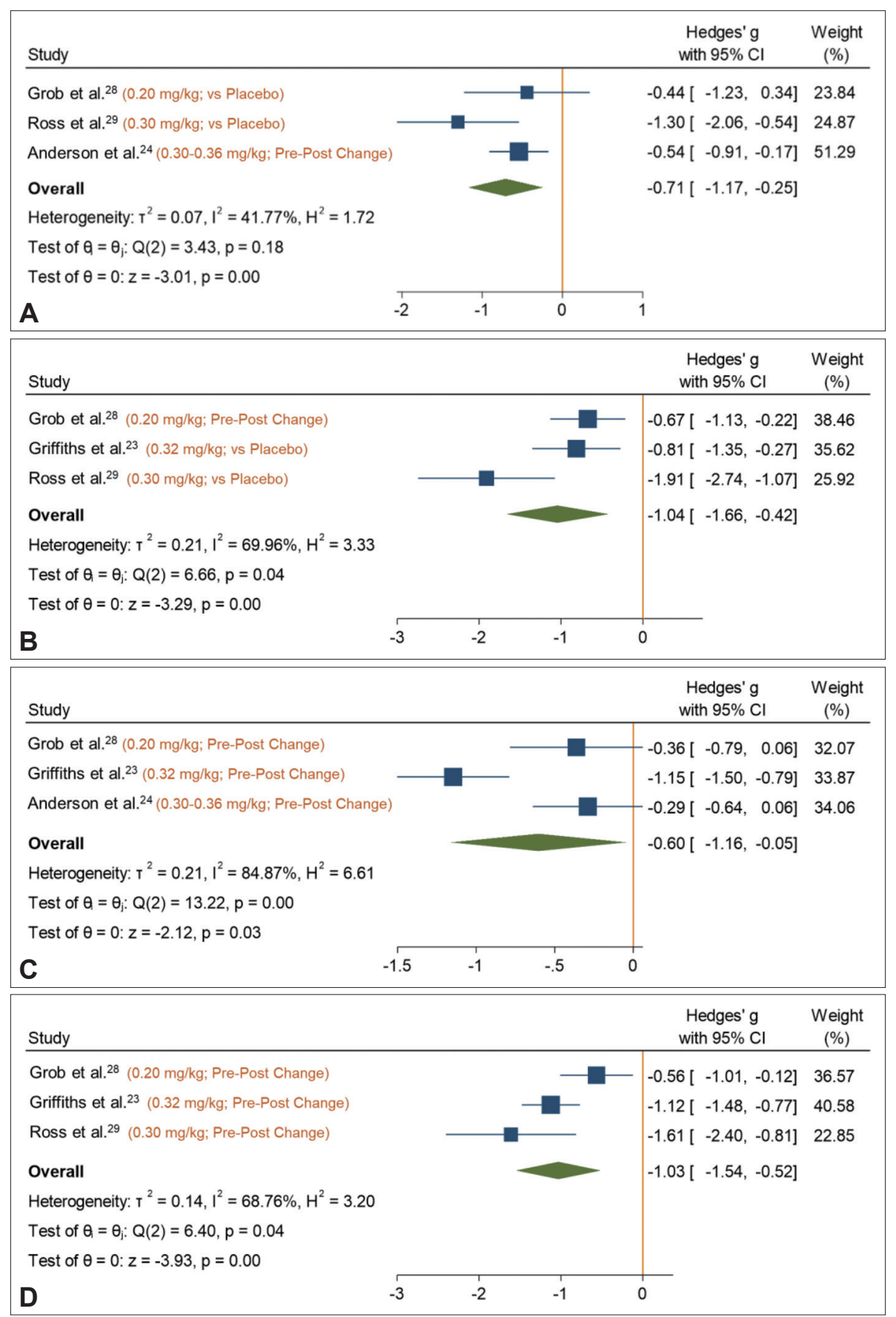

Figure 2. Changes in trait anxiety after psilocybin treatment. A: Day 1. B: Month 1. C: Month 3. D: Month 6.

on 5-HT2A receptors. ${ }^{34}$ Previous animal studies have reported the anxiolytic effect of 5-HT2A agonist, such as 2,5-dimethoxy4-iodoamphetamine, indicating its potentially moderating role in anxiety development though a brain network. ${ }^{35-37}$ However, little is known regarding the underlying biological mechanism of psilocybin's therapeutic effect in humans. A resting-state functional magnetic resonance imaging study demonstrated that psilocybin might decrease the cerebral blood flow to a significant magnitude over the medial prefrontal cortex (mPFC), an- terior cingulate cortex (ACC), and posterior cingulate cortex. ${ }^{38}$ Functional neuroimaging investigations revealed that anxiety levels were associated with the magnitude of responses to the stimuli presented in the mPFC and ACC. ${ }^{39,40}$ Another meta-analytic study provided convergent evidence that the ACC and mPFC significantly contributed to the onset of anxiety disorder by regulating emotions, affectional expression, and physiological reactions. ${ }^{41}$ Notably, the current study observed that the anxiolytic effects of psilocybin could be sustained up to 6 


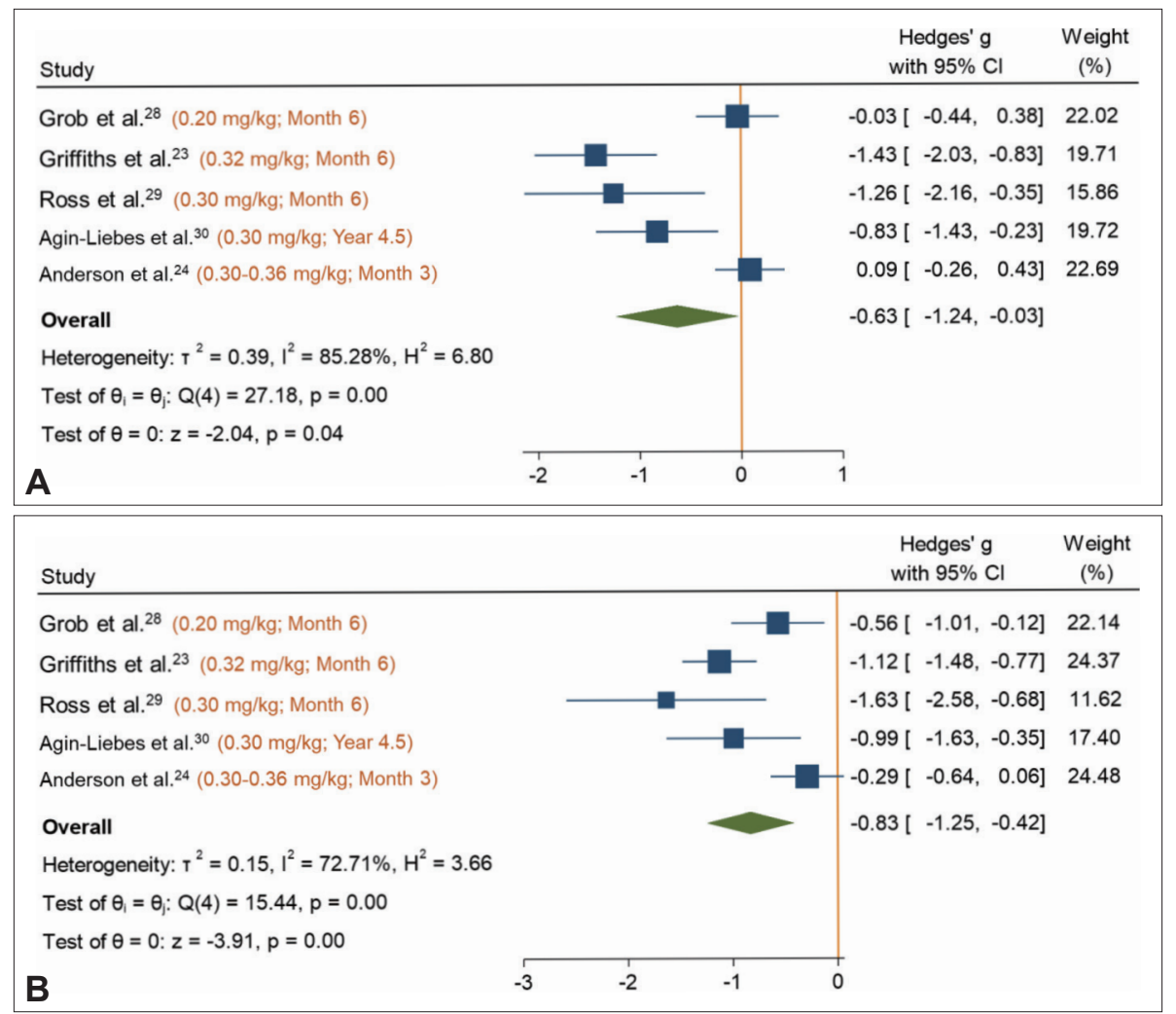

Figure 3. Pre-post changes in primary outcomes at last follow-up time point. A: State anxiety. B: Trait anxiety.

months after a single treatment dose. Therefore, psilocybin might exert therapeutic effects by destabilizing the local brain network hubs (e.g., ACC and mPFC) and global network connectivity by enhancing the neuronal avalanches, probably causing the "resetting" effect after the acute effects of treatment and altering the brain network activity for a long-term. ${ }^{42}$

\section{Tolerability of psilocybin and probability of abuse}

Regarding the rates of all-cause discontinuation and $\mathrm{HR}$, psilocybin is not different from placebo, suggesting that it is well tolerated by patients with advanced physical illnesses. However, psilocybin revealed significantly higher SBP and DBP than placebo during treatment session. Although these changes were transient and self-limited, it should be noticed that these temporal changes might result in harmful reactions in some patients with life-threatening illnesses. For instance, long-term AIDS survivors had a predominant burden of cardiovascular disease. ${ }^{43}$ Moreover, one of the trials included in the current study had reported higher rates of blood pressure among their participants. ${ }^{24}$

Nevertheless, in addition to the promising effectiveness of psilocybin, the possibility of its abuse has garnered attention. Although psilocybin has been reported to not induce dependence, craving, or withdrawal, ${ }^{44}$ the probability of its abuse cannot be neglected. A previous study revealed that psilocybin produced significantly higher potential abuse scores on the Addiction
Research Center Inventory amphetamine and lysergic acid diethylamide scales. ${ }^{17} \mathrm{~A}$ comprehensive review addressing medical psilocybin suggested that psilocybin has an abuse potential requiring appropriate scheduling if approved as medicine; however, it can provide therapeutic benefits with manageable adverse effects. ${ }^{45}$ Therefore, considering its excellent effectiveness of psilocybin in patients with life-threatening illnesses, it could be prescribed cautiously along with adequate monitoring.

\section{Limitations}

This study had several limitations. First, the power of analysis of publication bias is limited owing to insufficient trials. We could identify only five studies meeting our inclusion criteria. Second, significant heterogeneity was observed regarding the effectiveness of psilocybin on state and trait anxiety, particularly for long-term effectiveness. Therefore, large-scale RCTs for validating the efficacy of psilocybin use for end-of-life anxiety symptoms are warranted. Third, the index population in the current study included those with life-threatening diseases, which might limit the generalizability of the current study. Finally, some studies used psychotherapy combined with psilocybin, ${ }^{24,29}$ and thereby psychotherapy might have contributed to the long-lasting effects of psilocybin.

In conclusion, the current study reported the promising effectiveness of psilocybin in treating end-of-life anxiety symp- 


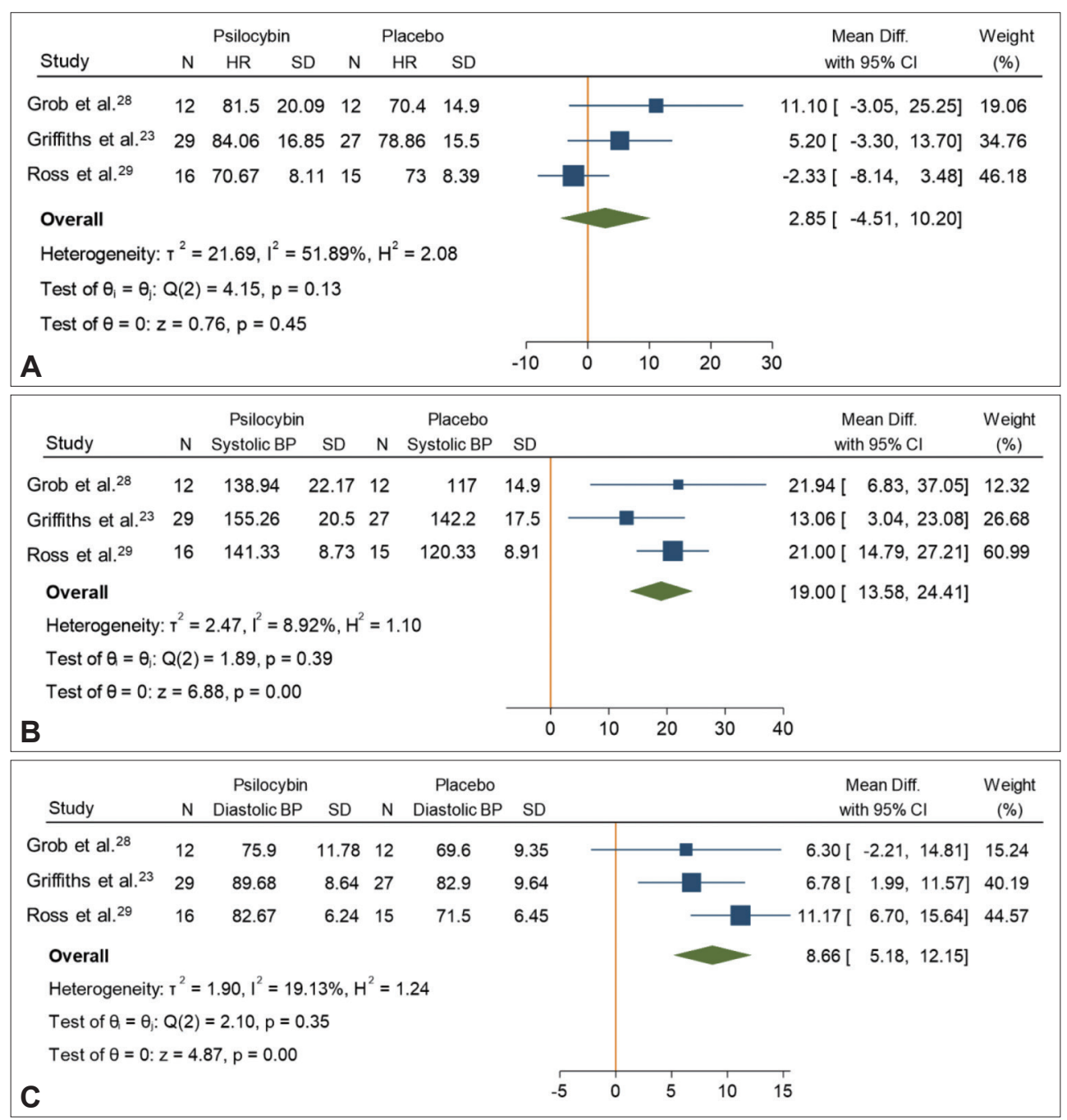

Figure 4. Peak heart rate and blood pressure after psilocybin treatment versus placebo. A: Heart rate. B: Systolic blood pressure. C: Diastolic blood pressure.

toms. Furthermore, it manifested the long-term effectiveness in trait anxiety after a single treatment dose, indicating its advantages in patients intolerable to severe adverse effects owing to their physical illnesses. Psilocybin could be prescribed cautiously along with adequate monitoring for the probability of potential abuse and transient changes in blood pressure during treatment. Because of the small sample sizes of the included studies and high heterogeneity on long-term outcomes, future randomized controlled trials with large sample sizes are needed. Clinicians should consider the clinical status of each patient before applying the current findings to clinical practice.

\section{Supplementary Materials}

The online-only Data Supplement is available with this article at https://doi.org/10.30773/pi.2021.0209.

\section{Availability of Data and Material}

The datasets generated or analyzed during the study are available from the corresponding author on reasonable request.

\section{Conflicts of Interest}

The authors have no potential conflicts of interest to disclose.

\section{Author Contributions}

Conceptualization: Dian-Jeng Li, Chih-Sung Liang. Data curation: Chia-Ling Yu, Fu-Chi Yang, Szu-Nian Yang, Ping-Tao Tseng, Brendon Stubbs, Ta-Chuan Yeh, Chih-Wei Hsu. Formal analysis: Dian-Jeng Li, Chih-Sung Liang. Investigation: Chia-Ling Yu, Fu-Chi Yang, Szu-Nian Yang, Ping-Tao Tseng. Methodology: Dian-Jeng Li, Chih-Sung Liang. Project administration: Dian-Jeng Li, Chih-Sung Liang. Writing_original draft: Chia-Ling Yu, Fu-Chi Yang. Writing_review \& editing: Szu-Nian Yang, Ping-Tao Tseng, Brendon Stubbs, Ta-Chuan Yeh, Chih-Wei Hsu, Dian-Jeng Li, Chih-Sung Liang.

\section{ORCID iDs}

Chia-Ling Yu Fu-Chi Yang Szu-Nian Yang

Ping-Tao Tseng Brendon Stubbs https://orcid.org/0000-0003-4471-8711 https://orcid.org/0000-0001-6831-3634 https://orcid.org/0000-0003-1960-1079 https://orcid.org/0000-0001-5761-7800 https://orcid.org/0000-0001-7387-3791 
Ta-Chuan Yeh Chih-Wei Hsu Dian-Jeng Li Chih-Sung Liang

https://orcid.org/0000-0002-1016-3634 https://orcid.org/0000-0002-8650-4060 https://orcid.org/0000-0002-6036-045X https://orcid.org/0000-0003-1138-5586

\section{Funding Statement}

None

\section{REFERENCES}

1. Antoni MH. Psychosocial intervention effects on adaptation, disease course and biobehavioral processes in cancer. Brain Behav Immun 2013;30(Suppl):S88-S98.

2. Gil F, Costa G, Hilker I, Benito L. First anxiety, afterwards depression: psychological distress in cancer patients at diagnosis and after medical treatment. Stress Health 2012;28:362-367.

3. Linden W, Vodermaier A, Mackenzie R, Greig D. Anxiety and depression after cancer diagnosis: prevalence rates by cancer type, gender, and age. J Affect Disord 2012;141:343-351.

4. Perez MN, Traino KA, Bakula DM, Sharkey CM, Espeleta HC, Delozier $\mathrm{AM}$, et al. Barriers to care in pediatric cancer: the role of illness uncertainty in relation to parent psychological distress. Psychooncology 2020; 29:304-310.

5. Vander Haegen M, Etienne AM. Intolerance of uncertainty in parents of childhood cancer survivors: a clinical profile analysis. J Psychosoc Oncol 2018;36:717-733

6. Sharpe L, Curran L, Butow P, Thewes B. Fear of cancer recurrence and death anxiety. Psychooncology 2018;27:2559-2565.

7. Antoni MH, Dhabhar FS. The impact of psychosocial stress and stress management on immune responses in patients with cancer. Cancer 2019;125:1417-1431

8. Antoni MH, Lutgendorf SK, Cole SW, Dhabhar FS, Sephton SE, McDonald PG, et al. The influence of bio-behavioural factors on tumour biology: pathways and mechanisms. Nat Rev Cancer 2006;6:240-248.

9. O’Brien KK, Bayoumi AM, Strike C, Young NL, Davis AM. Exploring disability from the perspective of adults living with HIV/AIDS: development of a conceptual framework. Health Qual Life Outcomes 2008;6:76.

10. O’Brien KK, Bayoumi AM, Strike C, Young NL, King K, Davis AM. How do existing HIV-specific instruments measure up? Evaluating the ability of instruments to describe disability experienced by adults living with HIV. Health Qual Life Outcomes 2010;8:88.

11. Logie C, Gadalla TM. Meta-analysis of health and demographic correlates of stigma towards people living with HIV. AIDS Care 2009;21:742753.

12. Basu S, Chwastiak LA, Bruce RD. Clinical management of depression and anxiety in HIV-infected adults. AIDS 2005;19:2057-2067.

13. Nanni MG, Caruso R, Mitchell AJ, Meggiolaro E, Grassi L. Depression in HIV infected patients: a review. Curr Psychiatry Rep 2015;17:530.

14. Houck MM, Siegel JA. Illicit Drugs. In: Houck MM, Siegel JA, Editors. Fundamentals of Forensic Science (Third Edition). San Diego: Academic Press; 2015, p.315-352.

15. Vollenweider FX, Kometer M. The neurobiology of psychedelic drugs: implications for the treatment of mood disorders. Nat Rev Neurosci 2010;11:642-651.

16. Griffiths R, Richards W, Johnson M, McCann U, Jesse R. Mystical-type experiences occasioned by psilocybin mediate the attribution of personal meaning and spiritual significance 14 months later. J Psychopharmacol 2008;22:621-632.

17. Griffiths RR, Johnson MW, Richards WA, Richards BD, McCann U, Jesse R. Psilocybin occasioned mystical-type experiences: immediate and persisting dose-related effects. Psychopharmacology (Berl) 2011; 218:649-665.

18. Johnson MW, Griffiths RR. Potential therapeutic effects of psilocybin. Neurotherapeutics 2017;14:734-740.

19. de Veen BT, Schellekens AF, Verheij MM, Homberg JR. Psilocybin for treating substance use disorders? Expert Rev Neurother 2017;17:203212.

20. Grassi L, Caruso R, Hammelef K, Nanni MG, Riba M. Efficacy and safety of pharmacotherapy in cancer-related psychiatric disorders across the trajectory of cancer care: a review. Int Rev Psychiatry 2014;26:44-62.

21. Heissel A, Zech P, Rapp MA, Schuch FB, Lawrence JB, Kangas M, et al. Effects of exercise on depression and anxiety in persons living with HIV: a meta-analysis. J Psychosom Res 2019;126:109823.

22. Breitbart W, Rosenfeld B, Gibson C, Pessin H, Poppito S, Nelson C, Tomarken A, et al. Meaning-centered group psychotherapy for patients with advanced cancer: a pilot randomized controlled trial. Psychooncology 2010;19:21-28.

23. Griffiths RR, Johnson MW, Carducci MA, Umbricht A, Richards WA, Richards BD, et al. Psilocybin produces substantial and sustained decreases in depression and anxiety in patients with life-threatening cancer: a randomized double-blind trial. J Psychopharmacol 2016;30:11811197.

24. Anderson BT, Danforth A, Daroff PR, Stauffer C, Ekman E, Agin-Liebes $\mathrm{G}$, et al. Psilocybin-assisted group therapy for demoralized older long-term AIDS survivor men: an open-label safety and feasibility pilot study. EClinicalMedicine 2020;27:100538.

25. Horikawa M, Yagi A. The relationships among trait anxiety, state anxiety and the goal performance of penalty shoot-out by university soccer players. PLoS One 2012;7:e35727.

26. Sterne JAC, Savovic J, Page MJ, Elbers RG, Blencowe NS, Boutron I, et al. RoB 2: a revised tool for assessing risk of bias in randomised trials. BMJ 2019;366:14898.

27. Higgins JP, Thompson SG, Deeks JJ, Altman DG. Measuring inconsistency in meta-analyses. BMJ 2003;327:557-560.

28. Grob CS, Danforth AL, Chopra GS, Hagerty M, McKay CR, Halberstadt AL, et al. Pilot study of psilocybin treatment for anxiety in patients with advanced-stage cancer. Arch Gen Psychiatry 2011;68:71-78.

29. Ross S, Bossis A, Guss J, Agin-Liebes G, Malone T, Cohen B, et al. Rapid and sustained symptom reduction following psilocybin treatment for anxiety and depression in patients with life-threatening cancer: a randomized controlled trial. J Psychopharmacol 2016;30:1165-1180.

30. Agin-Liebes GI, Malone T, Yalch MM, Mennenga SE, Ponté KL, Guss J, et al. Long-term follow-up of psilocybin-assisted psychotherapy for psychiatric and existential distress in patients with life-threatening cancer. J Psychopharmacol 2020;34:155-166.

31. Onieva-Zafra MD, Fernandez-Munoz JJ, Fernandez-Martinez E, García-Sánchez FJ, Abreu-Sánchez A, Parra-Fernández ML. Anxiety, perceived stress and coping strategies in nursing students: a cross-sectional, correlational, descriptive study. BMC Med Educ 2020;20:370.

32. Silva AVD, Zandonade E, Amorim MHC. Anxiety and coping in women with breast cancer in chemotherapy. Rev Lat Am Enfermagem 2017; 25:e2891.

33. Erritzoe D, Roseman L, Nour MM, MacLean K, Kaelen M, Nutt DJ, et al. Effects of psilocybin therapy on personality structure. Acta Psychiatr Scand 2018;138:368-378.

34. Nichols DE. Psychedelics. Pharmacol Rev 2016;68:264-355.

35. Nic Dhonnchadha BA, Hascoet M, Jolliet P, Bourin M. Evidence for a 5-HT2A receptor mode of action in the anxiolytic-like properties of DOI in mice. Behav Brain Res 2003;147:175-184.

36. Ripoll N, Hascoet M, Bourin M. Implication of 5-HT2A subtype receptors in DOI activity in the four-plates test-retest paradigm in mice. Behav Brain Res 2006;166:131-139.

37. Ripoll N, Nic Dhonnchadha BA, Sebille V, Bourin M, Hascoët M. The four-plates test-retest paradigm to discriminate anxiolytic effects. Psychopharmacology (Berl) 2005;180:73-83.

38. Carhart-Harris RL, Erritzoe D, Williams T, Stone JM, Reed LJ, Colasanti A, et al. Neural correlates of the psychedelic state as determined by fMRI studies with psilocybin. Proc Natl Acad Sci U S A 2012;109: 2138-2143.

39. Simmons A, Matthews SC, Feinstein JS, Hitchcock C, Paulus MP, Stein 
MB. Anxiety vulnerability is associated with altered anterior cingulate response to an affective appraisal task. Neuroreport 2008;19:1033-1037.

40. Straube T, Schmidt S, Weiss T, Mentzel HJ, Miltner WH. Dynamic activation of the anterior cingulate cortex during anticipatory anxiety. Neuroimage 2009;44:975-981.

41. Shang J, Fu Y, Ren Z, Zhang T, Du M, Gong Q, et al. The common traits of the ACC and PFC in anxiety disorders in the DSM-5: meta-analysis of voxel-based morphometry studies. PLoS One 2014;9:e93432.

42. Nichols DE, Johnson MW, Nichols CD. Psychedelics as medicines: an emerging new paradigm. Clin Pharmacol Ther 2017;101:209-219.

43. Smit M, Brinkman K, Geerlings S, Smit C, Thyagarajan K, Sighem Av, et al. Future challenges for clinical care of an ageing population infected with HIV: a modelling study. Lancet Infect Dis 2015;15:810-818.

44. Aronson JK. Psilocybin. In: Aronson JK, Editor. Meyler's Side Effects of Drugs (Sixteenth Edition). Oxford: Elsevier; 2016, p.1048-1051.

45. Johnson MW, Griffiths RR, Hendricks PS, Henningfield JE. The abuse potential of medical psilocybin according to the 8 factors of the Controlled Substances Act. Neuropharmacology 2018;142:143-166. 
MEDLINE search strategy

1. Exp Depression/ or Depression.mp. or depress*.mp. (544253)

2. Exp Anxiety/ or Anxiety.mp. or anx*.mp. (253591)

3. Exp Psychological Distress/ or Psychological Distress.mp. (19853)

4. Exp Psilocybin/ or Psilocybin.mp. (1023)

5.1 or 2 or 3 (697862)

6.4 and 5 (188)

CENTRAL (Cochrane Library) search strategy

\#1. MeSH descriptor: [Psilocybin] explode all trees (70)

\#2. (Psilocybin):ti,ab,kw (Word variations have been searched) (155)

\#3. \#1 or \#2 (155)

Embase search strategy

\#1. 'psilocybin'/exp OR psilocybin $(1,886)$

\#2. 'depression'/exp OR depression $(752,352)$

\#3. 'anxiety'/exp OR 'anxiety' $(393,513)$

\#4. 'psychological distress assessment'/exp OR 'psychological distress assessment' (6346) \#5. \#2 OR \#3 OR \#4 $(943,976)$

\#6. \#1 AND \#5 (454)

PsycINFO search strategy

1. Exp Depression/ or Depression.mp. or depress*.mp. (375536)

2. Exp Anxiety/ or Anxiety.mp. or anx*.mp. (256781)

3. Exp Psychological Distress/ or Psychological Distress.mp. (20136)

4. Exp Psilocybin/ or Psilocybin.mp. (538)

5. 1 or 2 or 3 (525217)

6.4 and 5 (154) 


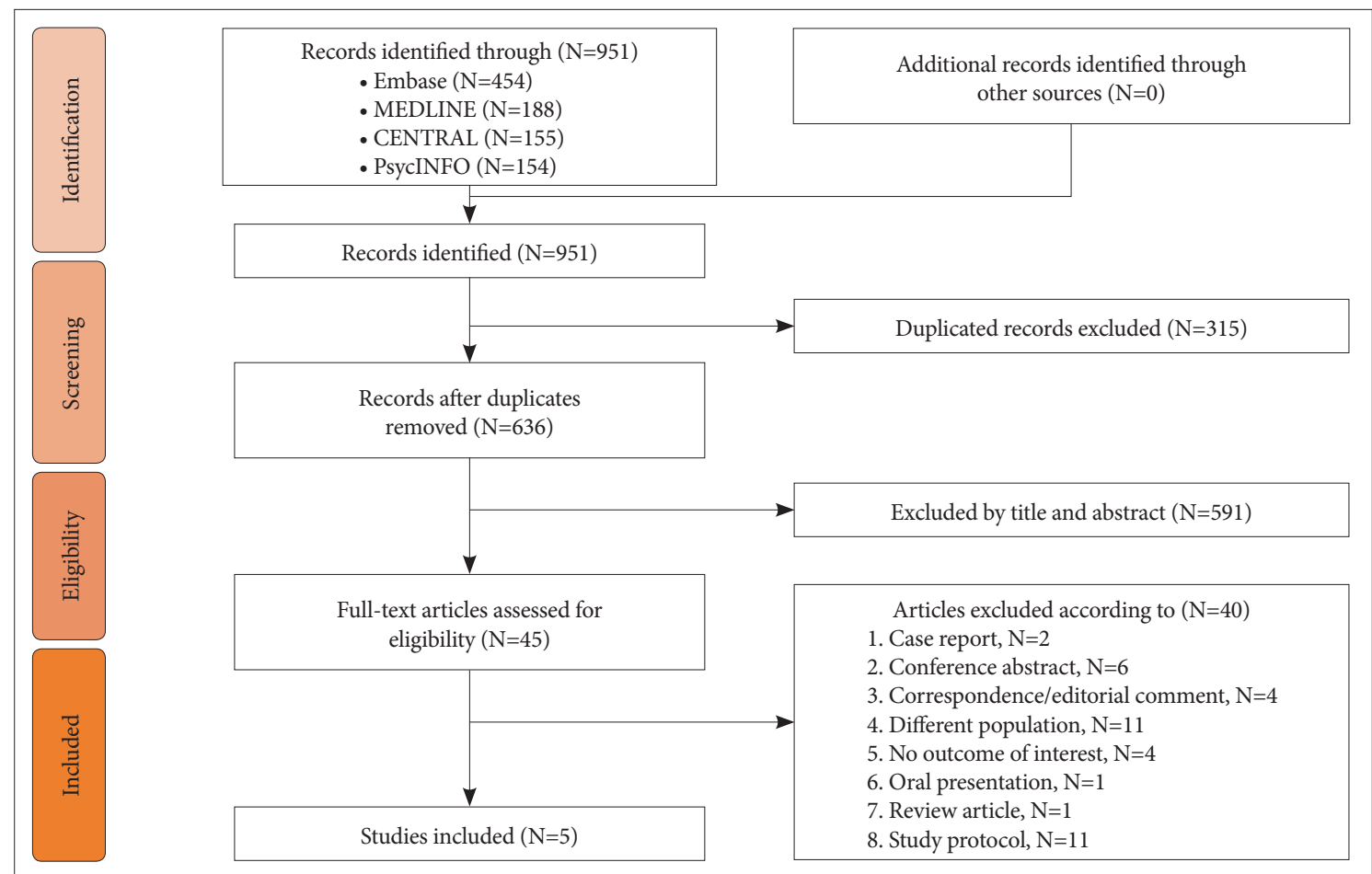

Supplementary Figure 1. PRISMA flow chart. 


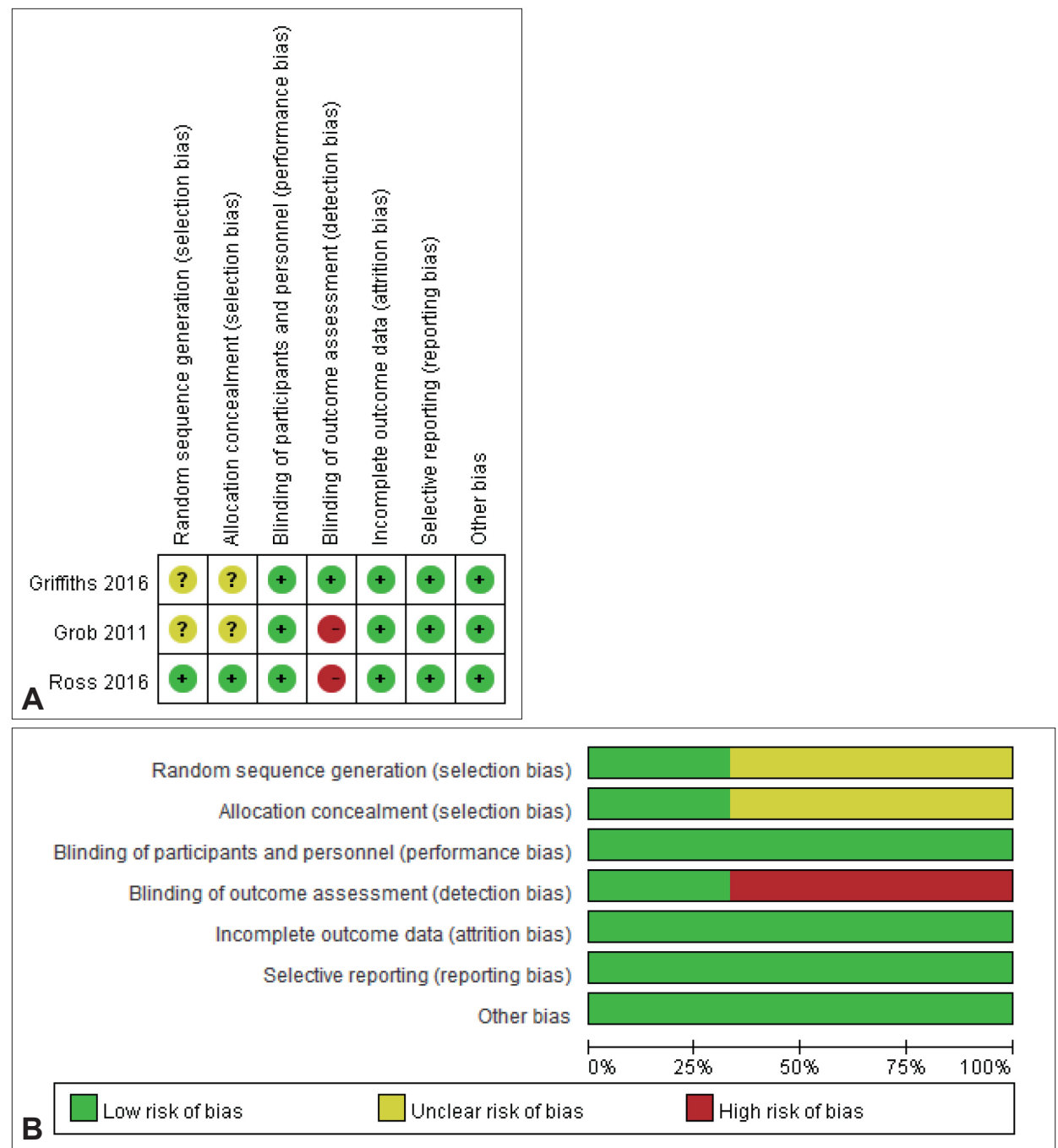

Supplementary Figure 2. Risk of bias for included randomized controlled trials. A: Risk of bias summary. B: Risk of bias graph. 


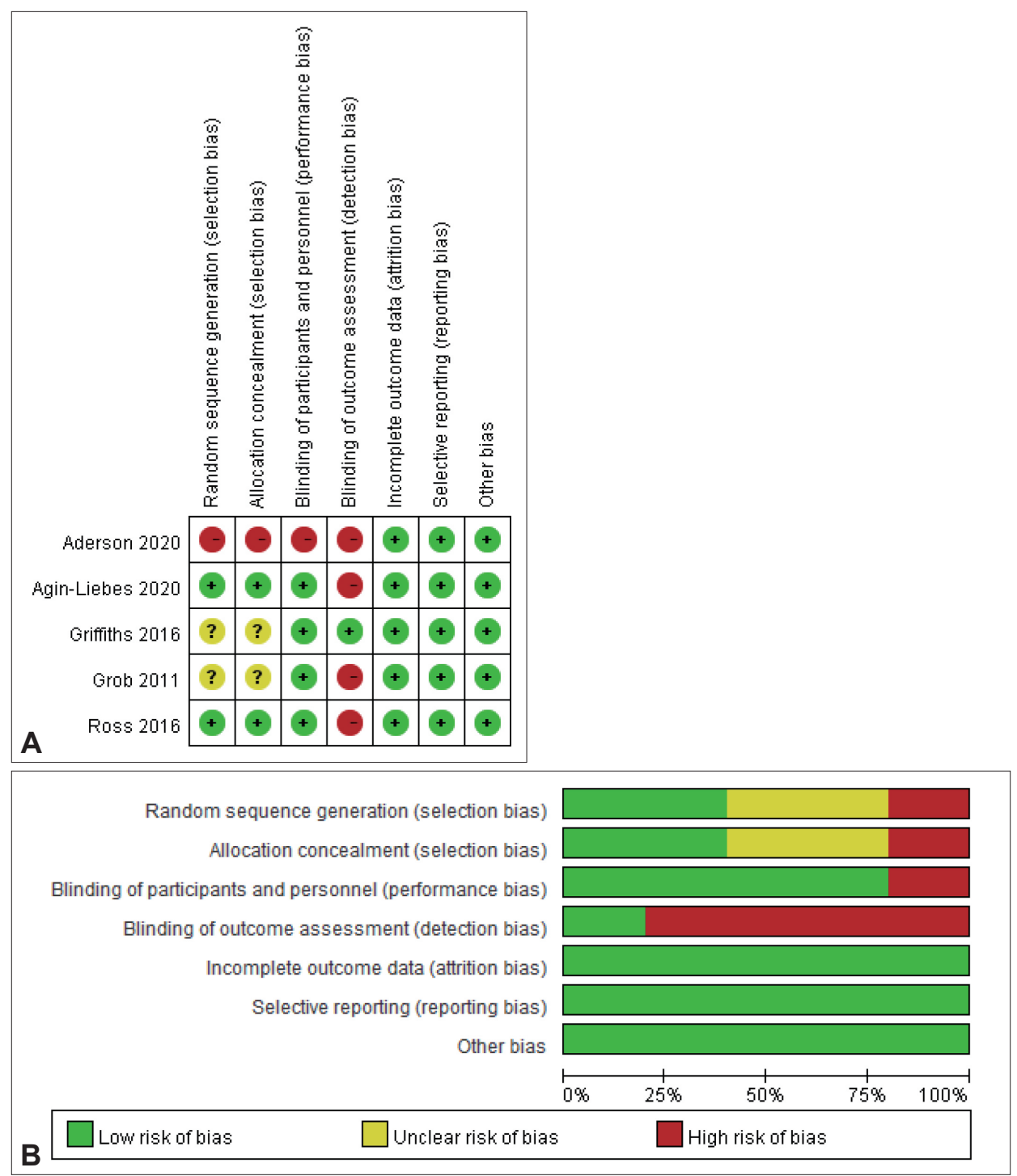

Supplementary Figure 3. Risk of bias for all included studies. A: Risk of bias summary. B: Risk of bias graph. 


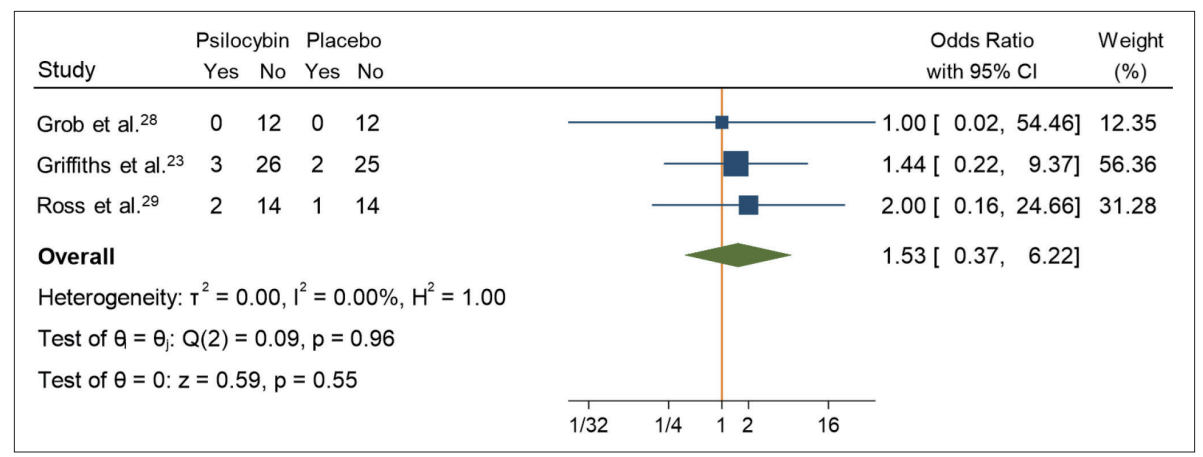

Supplementary Figure 4. All-cause discontinuation of psilocybin versus placebo. 


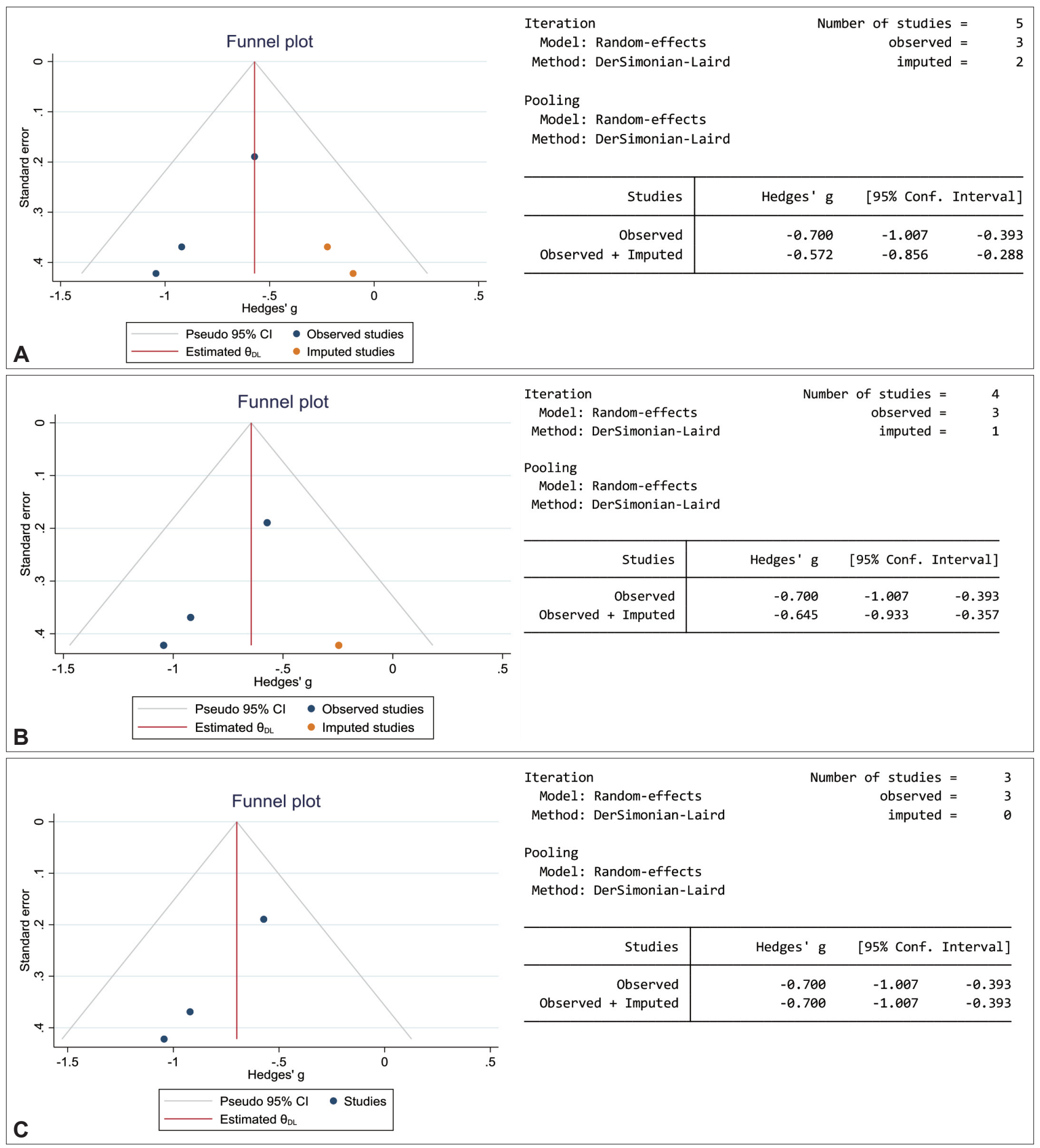

Supplementary Figure 5. Publication bias for state anxiety: 1 day after psilocybin treatment. A: Linear estimator. B: Run estimator. C: Quadratic estimator. 


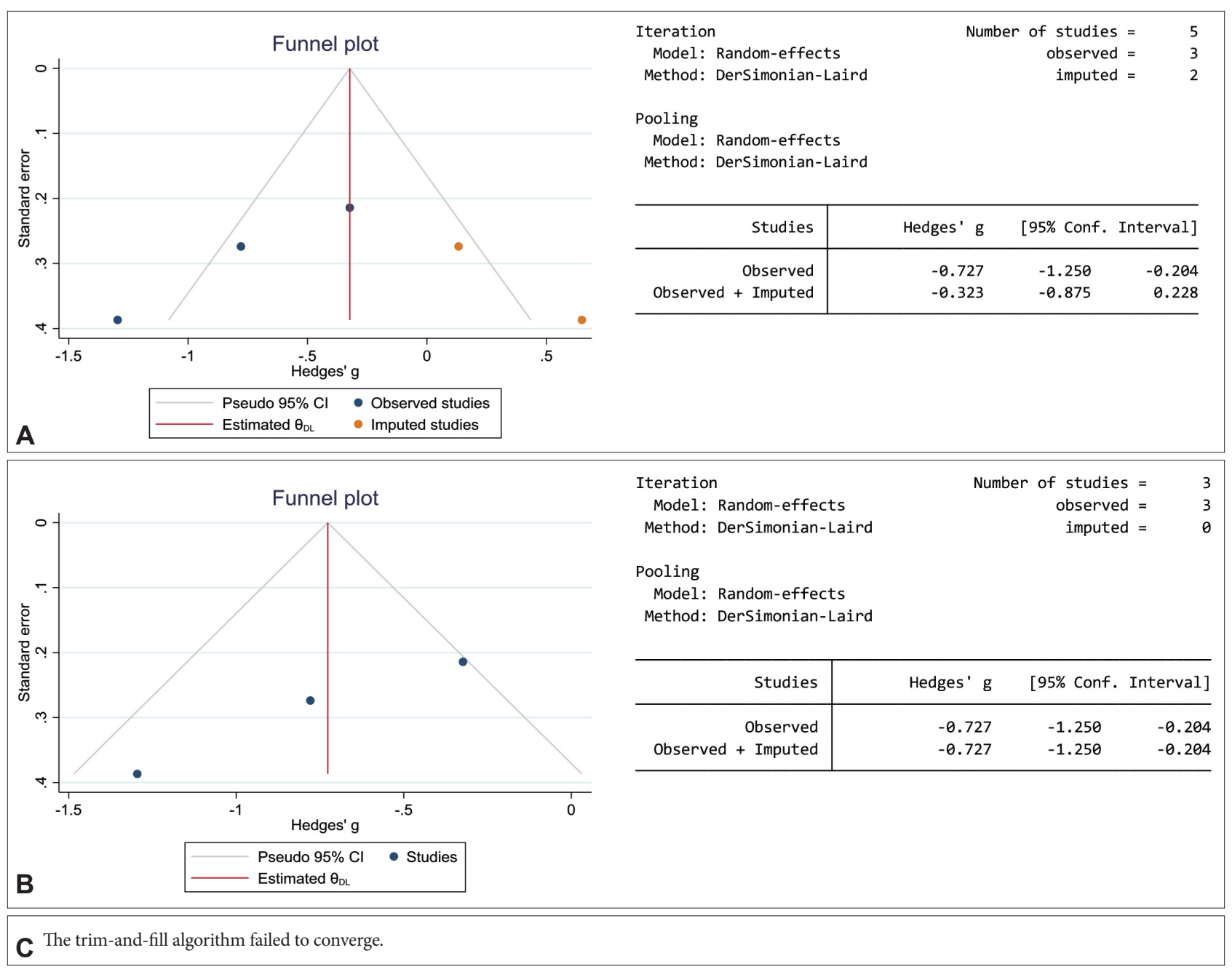

Supplementary Figure 6. Publication bias for state anxiety: 1 month after psilocybin treatment. A: Linear estimator. B: Run estimator. C: Quadratic estimator. 


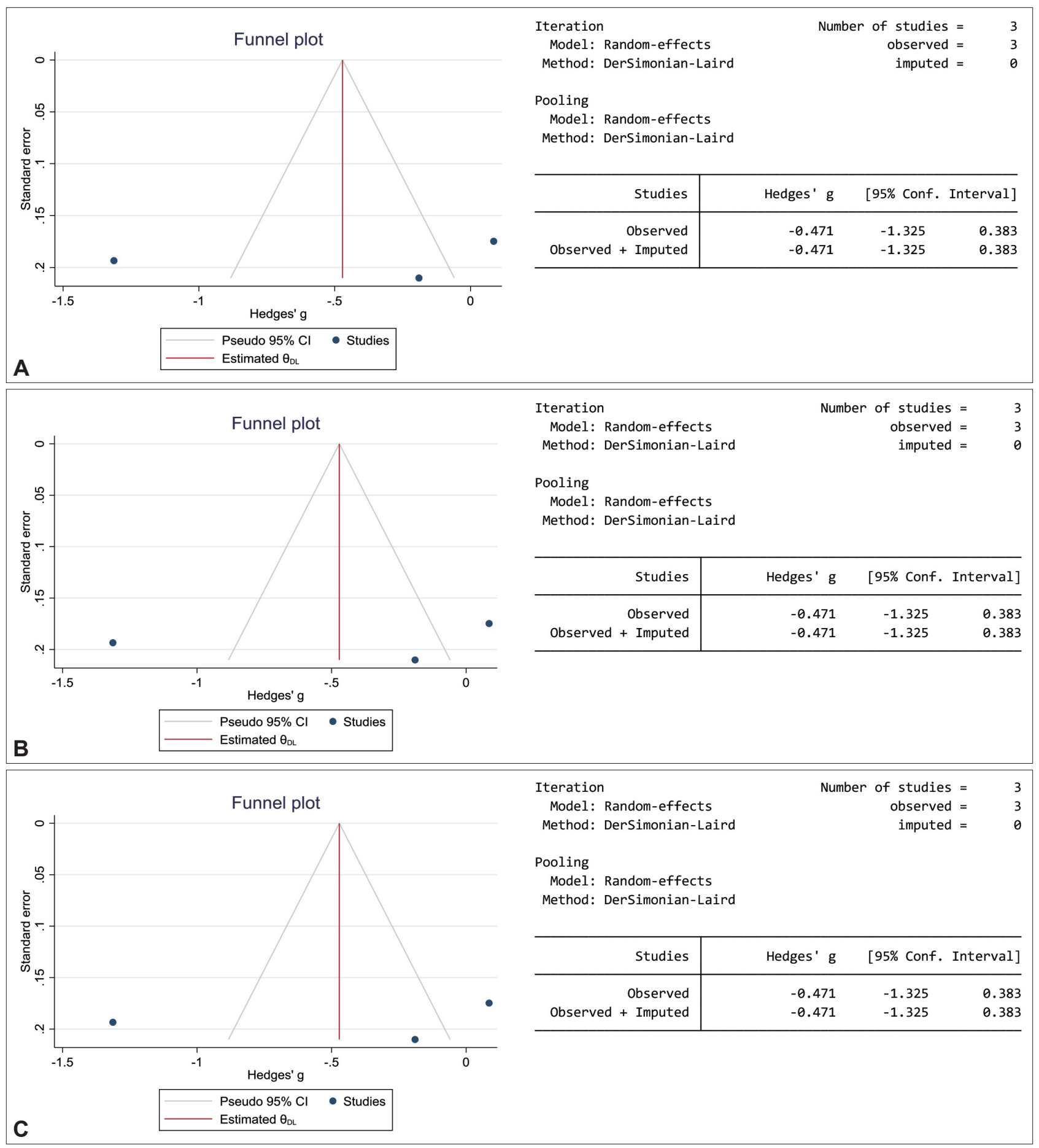

Supplementary Figure 7. Publication bias for state anxiety: 3 months after psilocybin treatment. A: Linear estimator. B: Run estimator. C: Quadratic estimator. 


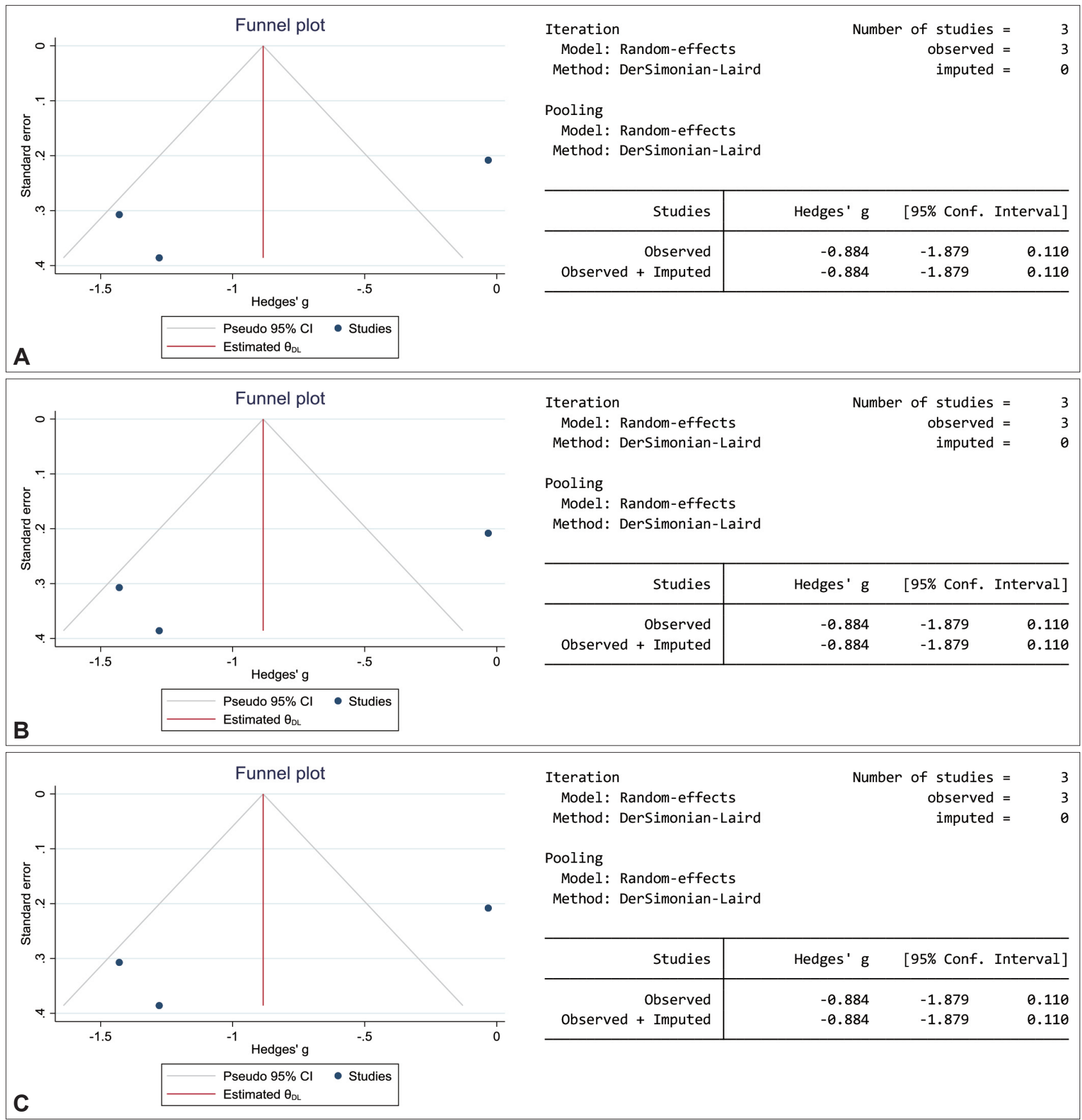

Supplementary Figure 8. Publication bias for state anxiety: 6 months after psilocybin treatment. A: Linear estimator. B: Run estimator. C: Quadratic estimator. 


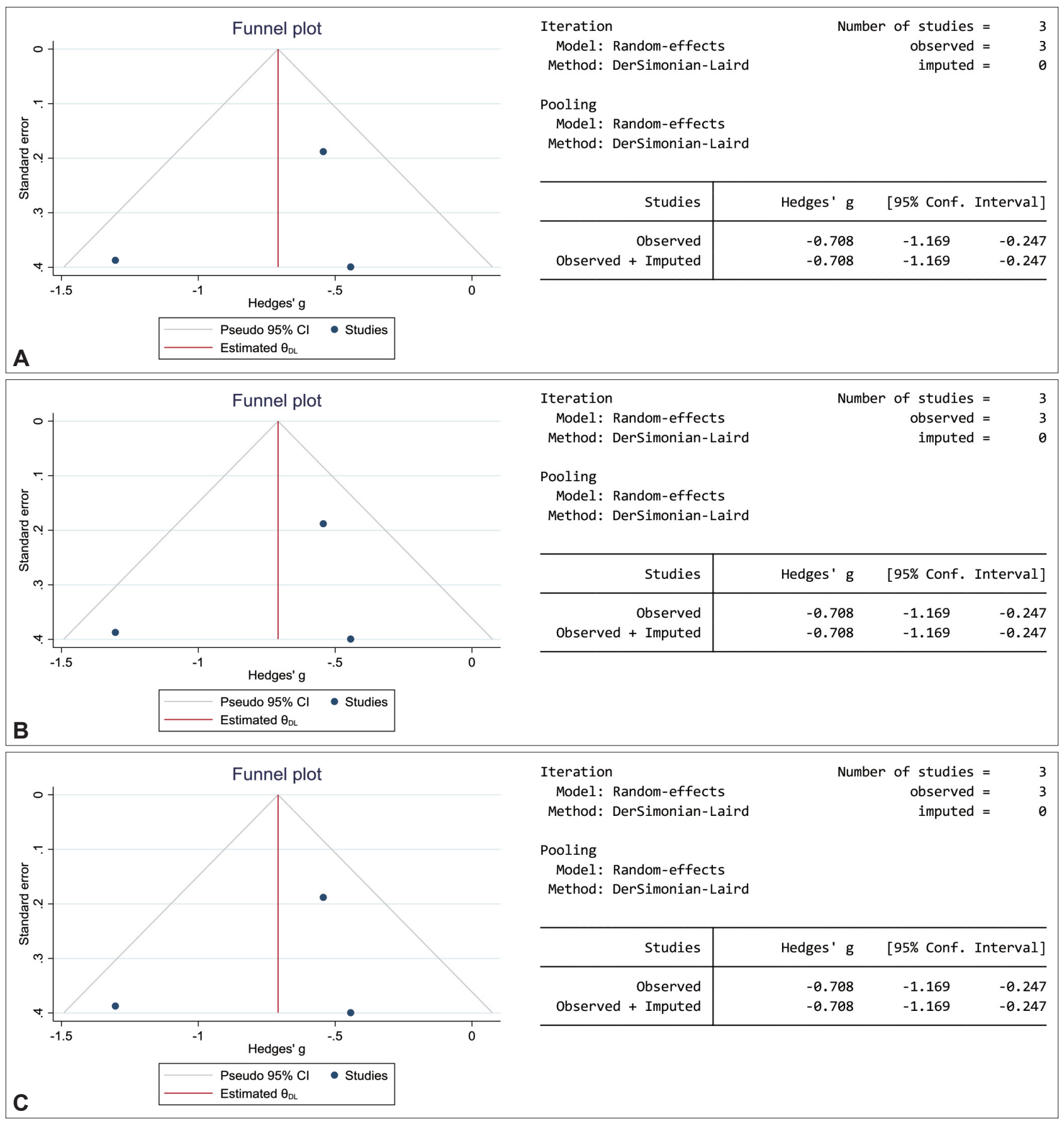

Supplementary Figure 9. Publication bias for trait anxiety: 1 day after psilocybin treatment. A: Linear estimator. B: Run estimator. C: Quadratic estimator. 


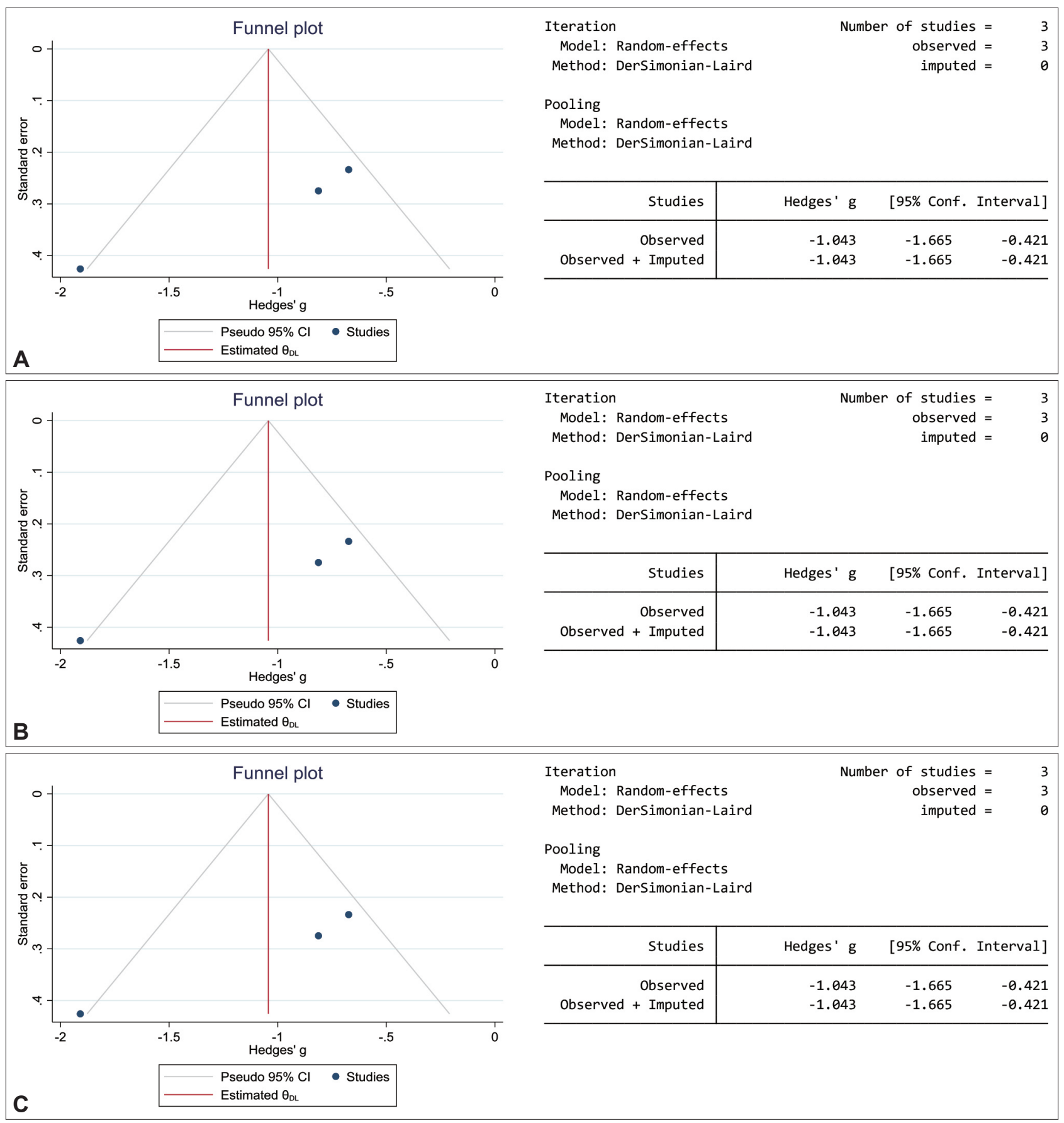

Supplementary Figure 10. Publication bias for trait anxiety: 1 month after psilocybin treatment. A: Linear estimator. B: Run estimator. C: Quadratic estimator. 


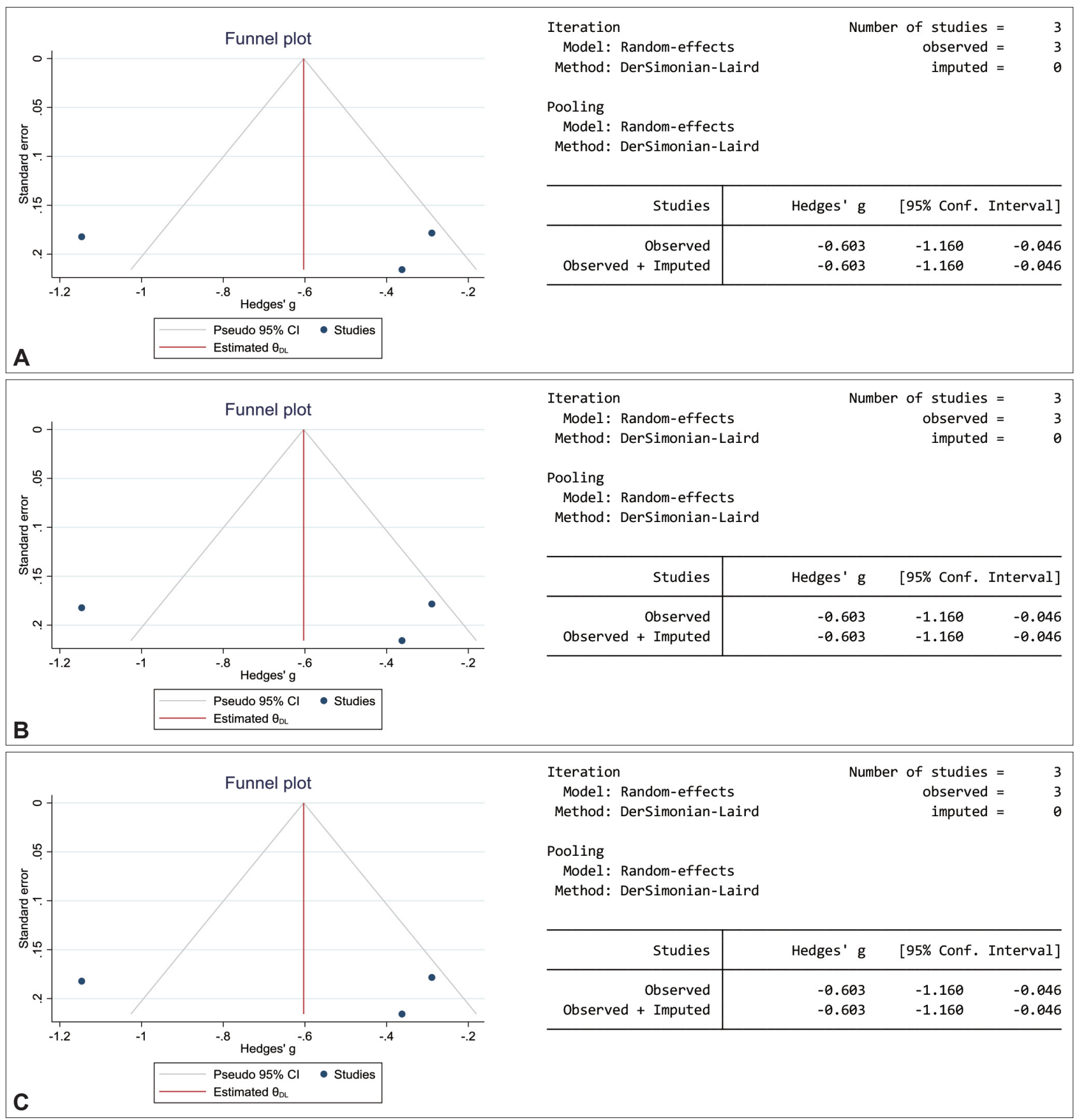

Supplementary Figure 11. Publication bias for trait anxiety: 3 months after psilocybin treatment. A: Linear estimator. B: Run estimator. C: Quadratic estimator. 


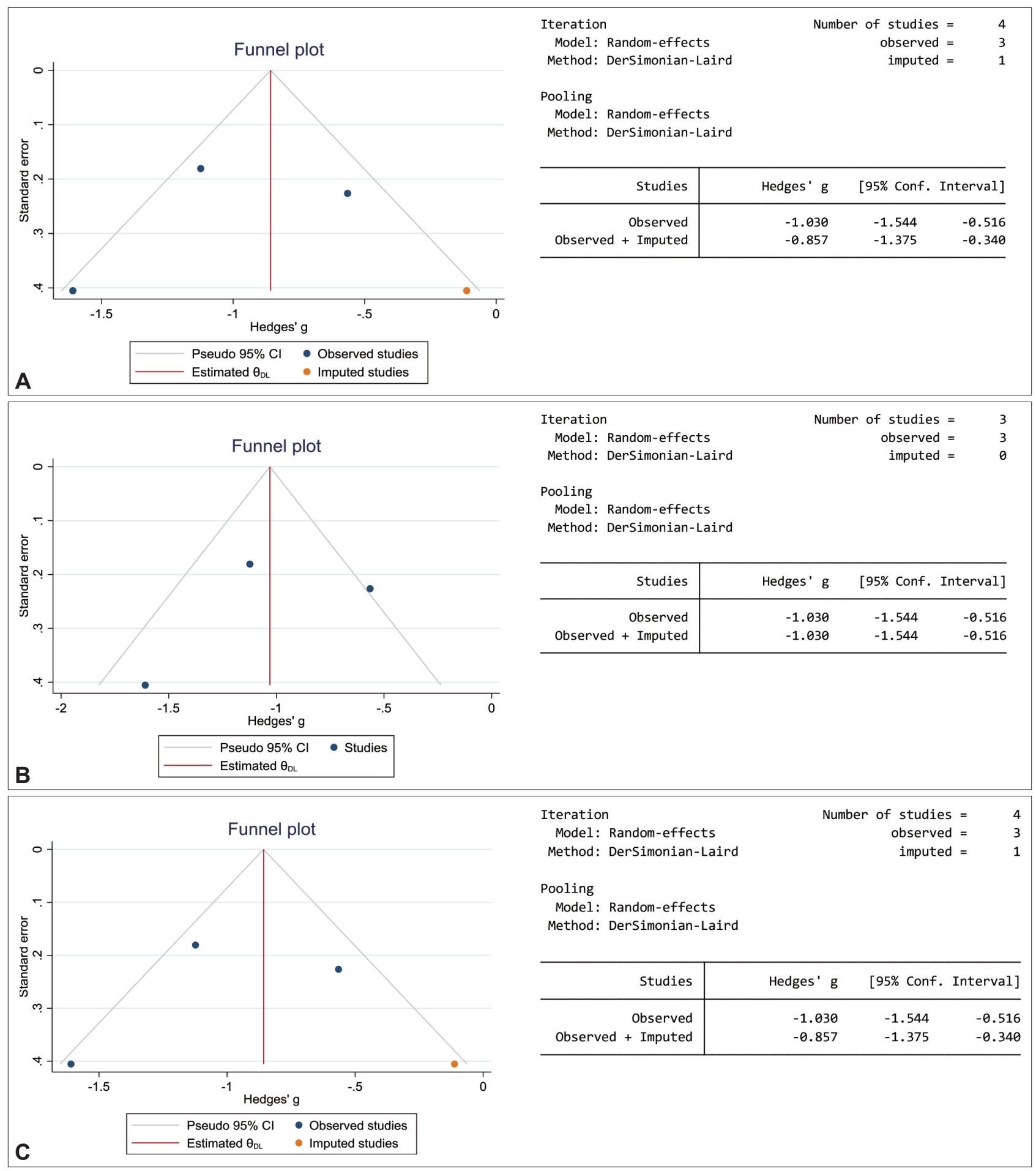

Supplementary Figure 12. Publication bias for trait anxiety: 6 months after psilocybin treatment. A: Linear estimator. B: Run estimator. C: Quadratic estimator. 


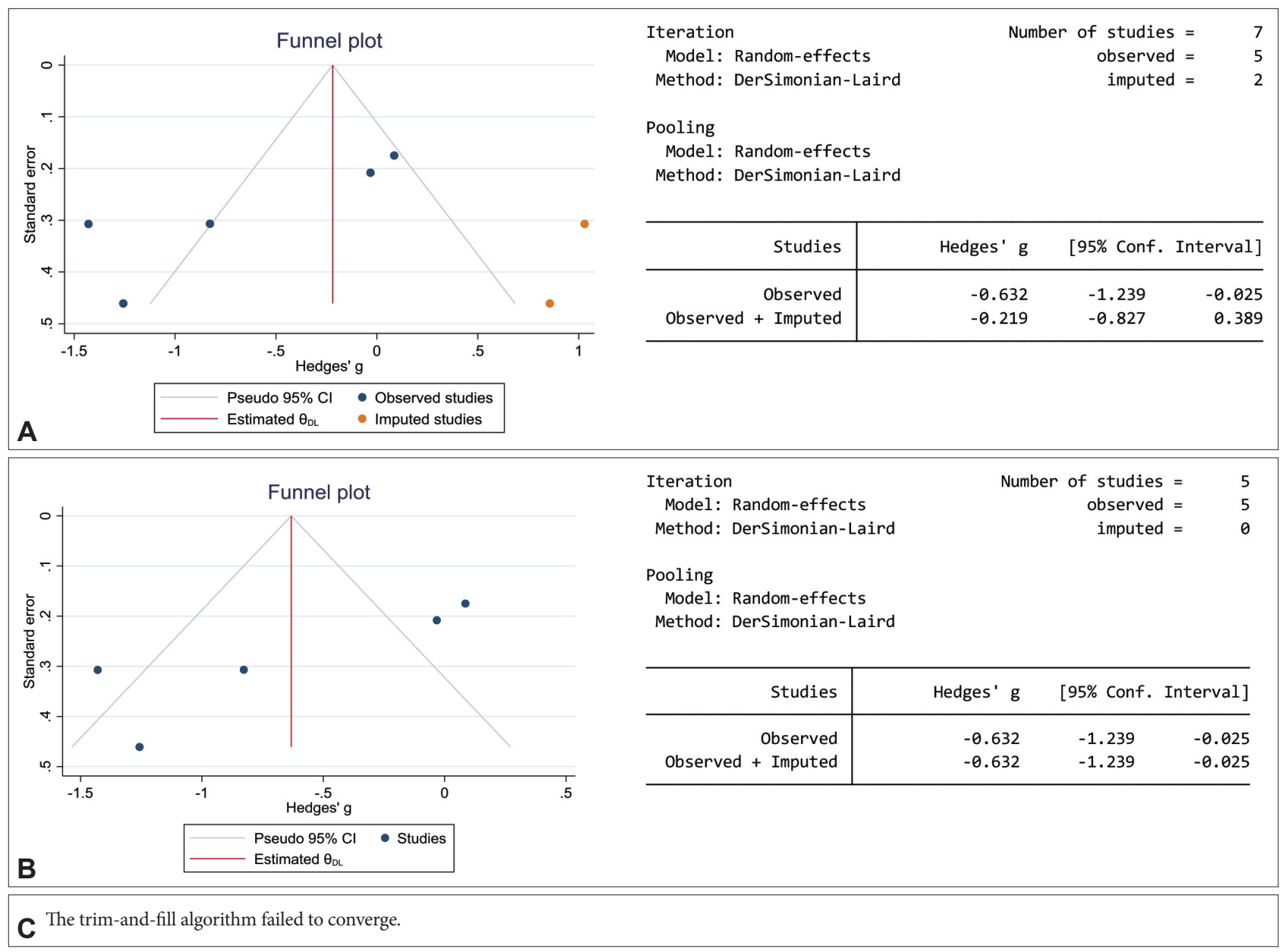

Supplementary Figure 13. Publication bias for state anxiety: Last follow-up time point for all included studies. A: Linear estimator. B: Run estimator. C: Quadratic estimator. 
A The trim-and-fill algorithm failed to converge.

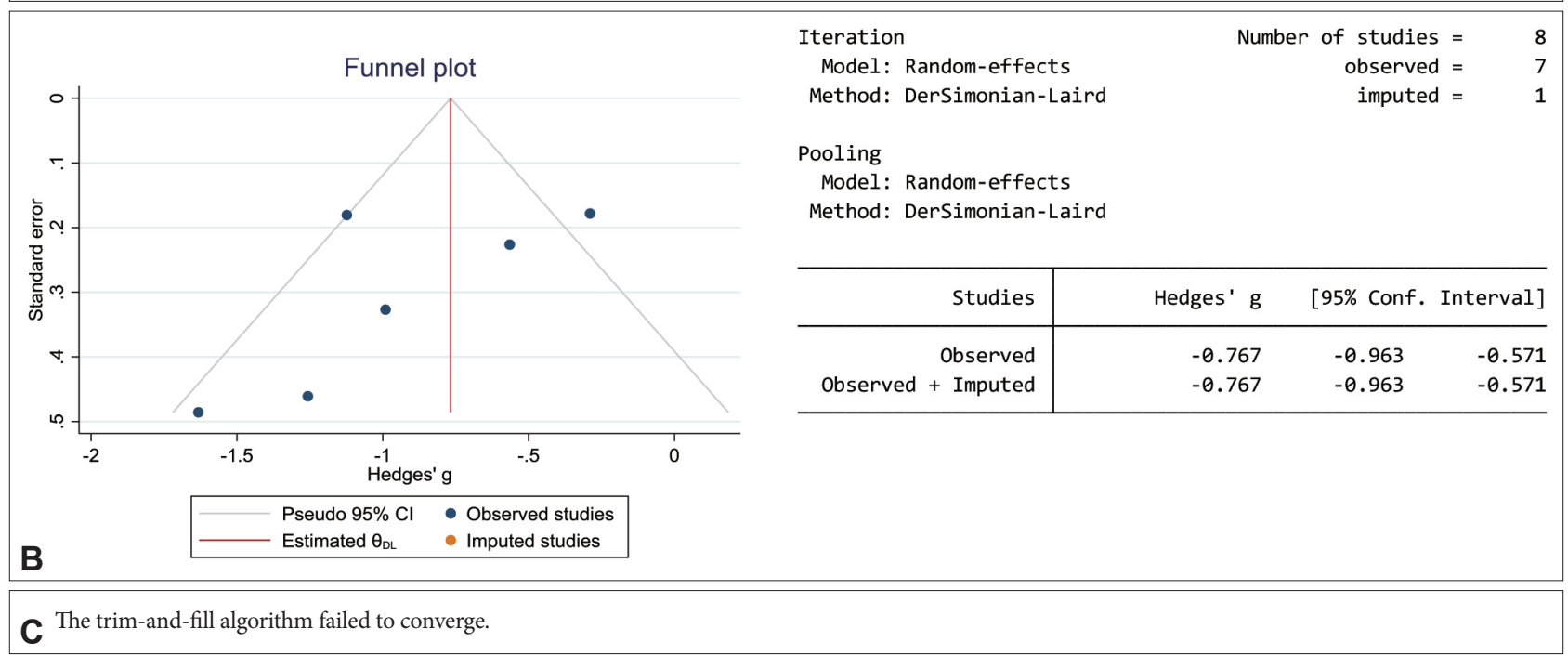

Supplementary Figure 14. Publication bias for trait anxiety: Last follow-up time point for all included studies. A: Linear estimator. B: Run estimator. C: Quadratic estimator. 